\title{
ON THE ASYMPTOTICS OF DETERMINANT OF LAPLACIAN AT THE PRINCIPAL BOUNDARY OF THE PRINCIPAL STRATUM OF THE MODULI SPACE OF ABELIAN DIFFERENTIALS
}

\author{
A. KOKOTOV
}

\begin{abstract}
Let $\mathcal{X}$ be a translation surface of genus $g>1$ with $2 g-2$ conical points of angle $4 \pi$ and let $\gamma, \gamma^{\prime}$ be two homologous saddle connections of length $s$ joining two conical points of $\mathcal{X}$ and bounding two surfaces $S^{+}$and $S^{-}$with boundaries $\partial S^{+}=\gamma-\gamma^{\prime}$ and $\partial S^{-}=\gamma^{\prime}-\gamma$. Gluing the opposite sides of the boundary of each surface $S^{+}, S^{-}$one gets two (closed) translation surfaces $\mathcal{X}^{+}, \mathcal{X}^{-}$of genera $g^{+}, g^{-} ; g^{+}+g^{-}=g$. Let $\Delta, \Delta^{+}$and $\Delta^{-}$be the Friedrichs extensions of the Laplacians corresponding to the (flat conical) metrics on $\mathcal{X}, \mathcal{X}^{+}$and $\mathcal{X}^{-}$respectively. We study the asymptotical behavior of the (modified, i.e. with zero modes excluded) zeta-regularized determinant $\operatorname{det}^{*} \Delta$ as $\gamma$ and $\gamma^{\prime}$ shrink. We find the asymptotics

$$
\operatorname{det}^{*} \Delta \sim \kappa s^{1 / 2} \frac{\operatorname{Area}(\mathcal{X})}{\operatorname{Area}\left(\mathcal{X}^{+}\right) \operatorname{Area}\left(\mathcal{X}^{-}\right)} \operatorname{det}^{*} \Delta^{+} \operatorname{det}^{*} \Delta^{-}
$$

as $s \rightarrow 0$; here $\kappa$ is a certain absolute constant admitting an explicit expression through spectral characteristics of some model operators. We use the obtained result to fix an undetermined constant in the explicit formula for $\operatorname{det}^{*} \Delta$ found in an earlier work by the author and D. Korotkin.
\end{abstract}

\section{INTRODUCTION}

Let $H_{g}(1, \ldots, 1)(2 g-2$ units $)$ be the principal stratum of the moduli space of Abelian differentials over compact Riemann surfaces of genus g. One defines $H_{g}(1, \ldots, 1)$ as the moduli space of pairs $(\mathcal{X}, \omega)$, where $\mathcal{X}$ is a compact Riemann surface of genus $g$ and $\omega$ is a holomorphic one-form (an Abelian differential) on $\mathcal{X}$ with $2 g-2$ zeros of multiplicity one. It is known $([13])$ that $H_{g}(1, \ldots, 1)$ is a connected complex orbifold of (complex) dimension $4 g-3$.

Let a pair $(\mathcal{X}, \omega)$ belong to $H_{g}(1, \ldots, 1)$. The holomorphic differential $\omega$ defines the conformal flat conical metric $|\omega|^{2}$ on $\mathcal{X}$. This metric has conical points of angle $4 \pi$ at the zeros of $\omega$ and trivial monodromy along any closed loop in $\mathcal{X} \backslash$ $\{$ conical points $\}$. Thus, the 2 -d manifold $\mathcal{X}$ equipped with metric $|\omega|^{2}$ becomes a so-called translation surface.

To the metric $|\omega|^{2}$ one can associate the (positive) Laplace operator $\Delta^{|\omega|^{2}}$ (often denoted below simply by $\Delta)$ with domain $C_{c}^{\infty}(\mathcal{X} \backslash\{$ conical points $\})$. The Friedrichs extension of $\Delta$ (from now on the notation $\Delta$ refers only to this selfadjoint operator

Received by the editors July 6, 2010.

2010 Mathematics Subject Classification. Primary 58J52; Secondary 32G15, 14H15, 30F10. 
in the Hilbert space $\left.L_{2}\left(\mathcal{X},|\omega|^{2}\right)\right)$ is known to have a discrete spectrum $0=\lambda_{0}<$ $\lambda_{1} \leq \lambda_{2} \leq \ldots$ of finite multiplicity. The operator zeta-function, defined for $\Re t>1$ as

$$
\zeta_{\Delta}(t)=\sum_{j=1}^{\infty} \lambda_{j}^{-t}
$$

admits analytic continuation to $\mathbb{C}$ as a meromorphic function with the only pole $t=1$. The (modified) zeta-regularized determinant of the operator $\Delta$ is defined via the relation $\log \operatorname{det}^{*} \Delta=-\zeta_{\Delta}^{\prime}(0)$.

If $\mathcal{X}$ is an elliptic curve, the Abelian differentials on $X$ have no zeros; the moduli space of Abelian differentials on Riemann surfaces of genus one is denoted by $H_{1}(\emptyset)$. Introduce the real-valued function $\mathcal{F}_{1}$ on $H_{1}(\emptyset)$ via

$$
H_{1}(\emptyset) \ni(\mathcal{X}, \omega) \mapsto \mathcal{F}_{1}(\mathcal{X}, \omega)=\operatorname{det}^{*} \Delta^{|\omega|^{2}} .
$$

In genus one the spectrum of the operator $\Delta$ is known explicitly and the direct calculation of the value $\zeta_{\Delta}^{\prime}(0)$ (which essentially reduces to making use of the first Kronecker limit formula) leads to the following expression (found in [20]; see also [19]):

$$
\mathcal{F}_{1}(\mathcal{X}, \omega)=\Im(B / A) \operatorname{Area}\left(\mathcal{X},|\omega|^{2}\right)|\eta(B / A)|^{4},
$$

where $A=\oint_{a} \omega, B=\oint_{b} \omega$ with $\{a, b\}$ being a canonical basis of cycles on $\mathcal{X}$, $\operatorname{Area}\left(\mathcal{X},|w|^{2}\right)=|\Im(A \bar{B})|$, and $\eta$ is the Dedekind eta-function

$$
\eta(\sigma)=\exp \left(\frac{\pi i \sigma}{12}\right) \prod_{n \in \mathbb{N}}(1-\exp (2 \pi i n \sigma)) .
$$

In [11 this classical result was generalized to the case of an arbitrary genus and an explicit expression for the function

$$
H_{g}(1, \ldots, 1) \ni(\mathcal{X}, \omega) \mapsto \mathcal{F}_{g}(\mathcal{X}, \omega)=\operatorname{det}^{*} \Delta^{|\omega|^{2}}
$$

was found. To formulate this result we need to introduce some auxiliary objects. Let $\left\{a_{\alpha}, b_{\alpha}\right\}_{\alpha=1, \ldots, g}$ be a canonical basis of cycles on $\mathcal{X}$. Denote by $\widehat{\mathcal{X}}$ a fundamental polygon obtained via cutting the surface $\mathcal{X}$ along a system of $2 g$ loops starting at some chosen point of $\mathcal{X}$ and homologous to the basic cycles.

Introduce the basis of normalized Abelian differentials $\left\{v_{\alpha}\right\}$ on $\mathcal{X}$, the matrix of corresponding $b$-periods $\mathbb{B}=\left(\oint_{\beta_{\alpha}} v_{\beta}\right)$ and the vector of Riemann constants:

$$
K_{\alpha}^{P}=\frac{1}{2}+\frac{1}{2} \mathbb{B}_{\alpha \alpha}-\sum_{\beta=1, \beta \neq \alpha}^{g} \oint_{a_{\beta}}\left(v_{\beta} \int_{P}^{x} v_{\alpha}\right),
$$

where the interior integral is taken along a path which does not intersect $\partial \widehat{\mathcal{X}}$. Let $E(P, Q)$ be the Schottky-Klein prime form (see [7]).

As in 8 introduce

- the following holomorphic multi-valued $(g / 2,-g / 2)$-differential $\sigma(P, Q)$ :

$$
\sigma(P, Q)=\exp \left\{-\sum_{\alpha=1}^{g} \oint_{a_{\alpha}} v_{\alpha}(R) \log \frac{E(R, P)}{E(R, Q)}\right\} ;
$$


the right-hand side of (1.3) is a nonvanishing holomorphic $g / 2$-differential on $\widehat{\mathcal{X}}$ with respect to $P$ and a nonvanishing holomorphic $(-g / 2)$-differential with respect to $Q$;

- the following holomorphic multi-valued $g(1-g) / 2$-differential on $\mathcal{X}$ :

$$
\mathcal{C}(P)=\frac{1}{\mathcal{W}\left[v_{1}, \ldots, v_{g}\right](P)} \sum_{\alpha_{1}, \ldots, \alpha_{g}=1}^{g} \frac{\partial^{g} \Theta\left(K^{P}\right)}{\partial z_{\alpha_{1}} \ldots \partial z_{\alpha_{g}}} v_{\alpha_{1}} \ldots v_{\alpha_{g}}(P)
$$

where

$$
\mathcal{W}(P):=\operatorname{det}_{1 \leq \alpha, \beta \leq g}\left\|v_{\beta}^{(\alpha-1)}(P)\right\|
$$

is the Wronskian determinant of holomorphic differentials at the point $P$.

Let $(\omega)=\sum_{k=1}^{2 g-2} P_{k}$ be the divisor of the holomorphic differential $\omega$ and denote by $\mathcal{A}_{P}(\cdot)$ the Abel map with the base point $P$. Then one has the relation

$$
\mathcal{A}((\omega))+2 K^{P}+\mathbb{B} \mathbf{r}+\mathbf{q}=0
$$

with some integer vectors $\mathbf{r}$ and $\mathbf{q}$. Let us emphasize that vectors $\mathbf{r}, \mathbf{q}$ as well as the prime form and the differentials $C$ and $\sigma$ depend on the choice of the fundamental polygon $\widehat{\mathcal{X}}$.

Now we are able to formulate the result from [11. One has the relation

$$
\mathcal{F}_{g}(\mathcal{X}, \omega)=\delta_{g} \operatorname{det} \Im \mathbf{B} \operatorname{Area}\left(\mathcal{X},|\omega|^{2}\right)\left|\tau_{g}\left(\mathcal{X}, \omega,\left\{a_{\alpha}, b_{\alpha}\right\}\right)\right|^{2},
$$

where $\delta_{g}$ is a constant depending only on genus $g$ and $\tau_{g}\left(\mathcal{X}, \omega,\left\{a_{\alpha}, b_{\alpha}\right\}\right)$ is defined up to a unitary multiplicative factor (and not a choice of the fundamental polygon!) by the formula

$$
\tau_{g}^{-6}\left(\mathcal{X}, \omega,\left\{a_{\alpha}, b_{\alpha}\right\}\right)=e^{2 \pi i\left\langle\mathbf{r}, K^{P}\right\rangle} C^{-4}(P) \prod_{k=1}^{2 g-2} \sigma\left(P_{k}, P\right)\left\{E\left(P_{k}, P\right)\right\}^{(g-1)} .
$$

Here $P$ is an arbitrary point of $\mathcal{X}$ and the integer vector $\mathbf{r}$ is defined by (1.6), the values of the prime form and $\sigma$ at the zeros $P_{k}$ of the differential $\omega$ are calculated in the local parameter $x_{k}(Q)=\sqrt{\int_{P_{k}}^{Q} \omega}$, and the values of the prime form and $\sigma$ at the point $P$ are taken in the local parameter $z(Q)=\int^{Q} \omega$; the expression (1.8) is independent of the choice of $P$.

Remark 1 . In case $g=1$, using (1.1), the formula

$$
C(P)=2 \pi i \eta^{3}(B / A) e^{-\pi i \frac{B}{4 A}}
$$

from ([8], p. 21) and the relation $K^{P}=\frac{1}{2}+\frac{B}{2 A}$ (implying $\mathbf{r}=-1$ in (1.6) ) together with (1.7) and (1.8), one gets the relation

$$
\delta_{1}=(2 \pi)^{-4 / 3} .
$$

One of the main motivations of this paper is to fix the undetermined constant $\delta_{g}$ in (1.7) for $g>1$. To this end we are to study the asymptotics of $\operatorname{det}^{*} \Delta$ when two zeros of the differential collide and the surface $\mathcal{X}$ degenerates to a nodal surface with two irreducible components $\mathcal{X}^{+}$and $\mathcal{X}^{-}$.

In the terminology of [6] we approach the principal boundary of $H_{g}(1, \ldots, 1)$, shrinking two homologous saddle connections (i.e. geodesics, joining two colliding zeros). One can think about this situation as follows. Let $g^{+}, g^{-} \geq 1$ be integers 
such that $g^{+}+g^{-}=g$ and let $\left(\mathcal{X}^{ \pm}, \omega^{ \pm}\right) \in H_{g^{ \pm}}(1, \ldots, 1)\left(2 g^{ \pm}-2\right.$ units $)$. Introduce two straight cuts, $\left[P_{+}, P_{+}(s)\right]$ and $\left[P_{-}, P_{-}(s)\right]$, of equal length $s$ : one on the translation surface $\mathcal{X}^{+}$and another on the translation surface $\mathcal{X}^{-}$(these cuts should not contain the conical points). Identifying each shore of the cut on the surface $\mathcal{X}^{+}$with the corresponding shore of the cut on the surface $\mathcal{X}^{-}$, one gets a translation surface $\mathcal{X}^{(s)}$ of genus $g=g^{+}+g^{-}$with $2 g-2=\left(2 g^{+}-2\right)+\left(2 g^{-}-2\right)+2$ conical points of angle $4 \pi: 2 g^{+}-2$ of them, $P_{1}^{+}, \ldots, P_{g^{+}}^{+}$come from the surface $\mathcal{X}^{+}$, the $2 g^{-}-2$ points, $P_{1}^{-}, \ldots, P_{g^{-}}^{+}$, come from the surface $\mathcal{X}^{-}$and the remaining two conical points, $P_{r}$ and $P_{l}$, are the endpoints of the cuts. One can see that the points $P_{r}$ and $P_{l}$ are joined by two homologous saddle connections of length $s$ on the surface $\mathcal{X}^{(s)}$; these saddle connections are just the former shores of the cuts. The translation surface $\mathcal{X}^{(s)}$ comes with the holomorphic one-form $\omega^{(s)}$ having simple zeros at conical points of $\mathcal{X}^{(s)}$ and coinciding with $\omega^{ \pm}$in $\mathcal{X}^{ \pm} \backslash\left[P_{ \pm}, P_{ \pm}(s)\right] \subset \mathcal{X}^{(s)}$. So our goal is to study the asymptotics of $\operatorname{det}^{*} \Delta^{\left|\omega^{(s)}\right|^{2}}$ as $s \rightarrow 0$.

As we see, the degeneration scheme we encounter here is slightly different from the usual one (see, e.g., [7, [17, 27]), where the family of degenerating Riemann surfaces is obtained from two surfaces $\mathcal{X}^{+}$and $\mathcal{X}^{-}$via the well-known plumbing construction (one glues not the shores of the cuts as we do here but the annuli $A_{ \pm}=\left\{s \leq\left|z_{ \pm}\right| \leq 1\right\} \subset \mathcal{X}^{ \pm}$identifying the points $z_{+}$and $z_{-}$such that $\left.z_{+} z_{-}=s\right)$. Thus, one has to modify the results from ([7], 27]) concerning the asymptotical behavior of basic holomorphic objects on the degenerating Riemann surface (in particular those entering (1.8) ) in order to serve a different degeneration scheme. We do that in Section 2 where we closely follow Fay and Yamada (in fact we use a certain hybrid of their approaches). After this task is completed it becomes possible to calculate the asymptotics of $\tau_{g}\left(\mathcal{X}^{(s)}, \omega^{(s)},\left\{\alpha_{\alpha}, b_{\alpha}\right\}\right)$ from (1.8) as $s \rightarrow 0$. The result (obtained in subsection 2.4) looks as follows:

$$
\tau_{g}\left(\mathcal{X}^{(s)}, \omega^{(s)},\left\{\alpha_{\alpha}, b_{\alpha}\right\}\right) \sim \frac{1}{\sqrt{2}} s^{1 / 4} \tau_{g_{+}}\left(\mathcal{X}^{+}, \omega^{+},\left\{\alpha_{\alpha}^{+}, b_{\alpha}^{+}\right\}\right) \tau_{g_{-}}\left(\mathcal{X}^{-}, \omega^{-},\left\{\alpha_{\alpha}^{-}, b_{\alpha}^{-}\right\}\right)
$$

here the canonical basis $\left\{\alpha_{\alpha}, b_{\alpha}\right\}$ on the surface $\mathcal{X}^{(s)}$ is the union of the canonical basis $\left\{\alpha_{\alpha}^{+}, b_{\alpha}^{+}\right\}$on $\mathcal{X}^{+}$and the canonical basis $\left\{\alpha_{\alpha}^{-}, b_{\alpha}^{-}\right\}$on $\mathcal{X}^{-}$.

This result implies the asymptotics

$$
\operatorname{det}^{*} \Delta^{\left|\omega^{(s)}\right|^{2}} \sim \frac{\delta_{g}}{2 \delta_{g_{+}} \delta_{g_{-}}} s^{1 / 2} \frac{\operatorname{Area}(\mathcal{X})}{\operatorname{Area}\left(\mathcal{X}^{+}\right) \operatorname{Area}\left(\mathcal{X}^{-}\right)} \operatorname{det}^{*} \Delta^{+} \operatorname{det}^{*} \Delta^{-}
$$

and in order to fix the constant $\delta_{g}$ it is sufficient to obtain the asymptotics of $\operatorname{det}^{*} \Delta^{\left|\omega^{(s)}\right|^{2}}$ for some special elements $\left(\mathcal{X}_{0}^{ \pm}, \omega_{0}^{ \pm}\right)$of $H_{g_{ \pm}}(1, \ldots, 1)$ using another method and then compare the coefficients in the two asymptotics. (It should be noted that a similar program was recently realized by $\mathrm{R}$. Wentworth for the determinants of the Laplacian in the Arakelov metric in order to calculate the so-called bosonization constants (see 22]).) This is done in Section 3. The key idea (picked up by the author in a conversation with L. Hillairet) is the following: one can start (in the case of even genus $g=2 g_{0}$ ) with a translation surface $\mathcal{X}_{0}$ of genus $g_{0}$ with a cut $[P, P(s)]$ of length $s$ and glue two copies of $\mathcal{X}_{0}$ together along the cut. (So, one takes $\mathcal{X}^{+}=\mathcal{X}^{-}=\mathcal{X}_{0}$ in the above construction.) In this symmetric situation the Laplacian $\Delta$ on the translation surface $\mathcal{X}^{(s)}$ is unitarily equivalent to the direct 
sum of the two operators, $\Delta_{\mathcal{D}}$ and $\Delta_{\mathcal{N}}$, of Neumann and Dirichlet homogeneous boundary value problems in $\mathcal{X}_{0} \backslash[P, P(s)]$. Thus, one has the relation

$$
\operatorname{det}^{*} \Delta=\operatorname{det} \Delta_{\mathcal{D}} \operatorname{det}^{*} \Delta_{\mathcal{N}}
$$

(notice that the Dirichlet Laplacian has no zero modes and one does not modify its determinant here). It turns out that the asymptotics of $\operatorname{det} \Delta_{\mathcal{D}}$ and $\operatorname{det}^{*} \Delta_{\mathcal{N}}$ as $s \rightarrow 0$ can be found if one makes use of a certain variant of the BFK surgery formula (see [3]), the Wentworth lemma on the asymptotics of the Dirichlet-to-Neumann operator on a shrinking contour $([22])$ and a simple idea based on the rescaling properties of the determinant of the Laplacian. So, one can find the asymptotics $\operatorname{det}^{*} \Delta$ for a symmetric translation surface (and, therefore, for an arbitrary translation surface of genus which is an integer power of 2); a simple trick based on the BFK surgery formula reduces the general case to this symmetric one.

Beyond the scope of the present paper remains the case of another possible collision of conical points (in other words we consider here the asymptotical behavior of $\operatorname{det}^{*} \Delta$ only near a part of the principal boundary of the stratum): one can shrink a saddle connection of length $s \rightarrow 0$ which has no saddle connection homologous to it. In this case the underlying Riemann surface $\mathcal{X}^{(s)}$ does not degenerate (and tends to a nonsingular Riemann surface $\mathcal{X}^{(0)}$; we denote by $\Delta_{0}$ the Laplacian on the translation surface $\mathcal{X}^{(0)}$ ) but the colliding zeros form a single zero of multiplicity two (a conical point of the angle $6 \pi$ ). It is relatively easy to show that in this case the asymptotical behavior of $\tau_{g}$ has the form

$$
\tau_{g}\left(\mathcal{X}^{(s)}, \omega^{(s)},\left\{\alpha_{\alpha}, b_{\alpha}\right\}\right) \sim s^{1 / 36} \tilde{\tau}_{g}\left(\mathcal{X}^{(0)}, \omega_{0},\left\{\alpha_{\alpha}, b_{\alpha}\right\}\right),
$$

where $\tilde{\tau}_{g}$ is an analog of the function $\tau_{g}$ for the stratum $H_{g}(2,1, \ldots, 1)(2 g-4$ units; see [11] for definitions). This (together with results from [11]) leads to the asymptotics $\operatorname{det}^{*} \Delta \sim C_{g} s^{1 / 18} \operatorname{det}^{*} \Delta_{0}$ with the unknown constant $C_{g}$. Finding this constant presents an interesting open problem. Even more complicated looks the problem of finding the asymptotics of $\operatorname{det}^{*} \Delta$ at the boundary of a general stratum $H_{g}\left(k_{1}, \ldots, k_{M}\right)$; at the moment we see no reasonable approach to it.

Finally we notice that similar problems for a hyperbolic metric of constant curvature were studied by S. Wolpert ([26]) and R. Lundelius ([16]), and the case of the Arakelov metric (with curvature given by the Bergman 2-form) was considered in [10] and [24, 25] (the complete results were recently obtained by R. Wentworth in $[22]$.

The author is grateful to R. Wentworth for explaining to him some details from [22] and clarification of the reason for the divergence between the results of [7] and [27]; the author also thanks L. Hillairet for generous sharing of his ideas on the spectral theory of translation surfaces, D. Korotkin for numerous useful discussions and the anonymous referee for very useful comments and, in particular, for finding an extraneous factor in formula (3.33). The main part of this work was done during the stay of the author at the Max Planck Institute for Mathematics. The author thanks the institute for the hospitality and support. The research of the author was also supported by NSERC.

\section{FAmilies of DEgEnerating SURFACES AND ASYMPtoticAl FORMUlas}

We construct several one-parameter families of Riemann surfaces degenerating as the parameter tends to zero. 
Let $\mathcal{X}^{+}$and $\mathcal{X}^{-}$be two compact Riemann surfaces of genus $g^{+}$and $g^{-}, g^{ \pm} \geq 0$. Choose points $P_{ \pm} \in \mathcal{X}^{ \pm}$and their open neighborhoods $D^{ \pm} \subset \mathcal{X}^{ \pm}$such that for a certain choice of holomorphic local parameters $z^{ \pm}$on $\mathcal{X}^{ \pm}$one has $D^{ \pm}=\{P \in$ $\left.\mathcal{X}^{ \pm}:\left|z^{ \pm}(P)\right|<1\right\}$ and $z^{ \pm}\left(P^{ \pm}\right)=0$. Define the map $z: D^{+} \cup D^{-} \rightarrow \mathbb{C}$ setting $z(P)=z^{ \pm}(P)$ if $P \in D^{ \pm}$.

Using these data we construct three families of degenerating Riemann surfaces of genus $g^{-}+g^{+}$.

Case I. Let $s$ be a complex number, $|s|<1$ and let $P^{ \pm}(s)$ be the points in $D^{ \pm}$ such that $z\left(P^{ \pm}(s)\right)=s$.

Cut the discs $D^{ \pm}$along the (oriented) straight segments $\left[P^{ \pm}, P^{ \pm}(s)\right]$ and glue the surfaces $\mathcal{X}^{+}$and $\mathcal{X}^{-}$along these cuts identifying a point $P$ on the left shore of the "+"-cut with the point $Q\left(z^{+}(P)=z^{-}(Q)\right)$ on the right shore of the "-"cut and vice versa; the resulting topological real 2-d surface can be turned into a compact Riemann surface $\mathcal{X}_{s}$ of genus $g=g^{-}+g^{+}$in a usual way (one chooses the local parameter near the left endpoint $P$ of the cut as $\zeta(Q)=\sqrt{z(Q)}$, near the right endpoint $P(s)$ the local parameter is $\zeta(Q)=\sqrt{z(Q)-z(P(s))}$, and the choice of the local parameter at other points of $\mathcal{X}_{s}$ is obvious).

Case Ia. This family is constructed similarly to Case I, the only difference being the position of cuts inside the disks $D^{ \pm}$: choose a complex number $t,|t|<1$ and introduce the cuts inside the discs $D^{ \pm}$connecting the points $z=\sqrt{t}$ and $z=-\sqrt{t}$; after the same gluing of the shores of these cuts as in Case I we get the family $\mathcal{X}_{t}$ of degenerating compact Riemann surfaces.

Case Ib. This family is obtained similarly to Cases I and Ia, but instead of gluing the disks along the cuts we use the standard "plumbing construction" (see [7]). Choose $t,|t|<1$, delete from the discs $D^{ \pm}$the smaller discs $\left|z^{ \pm}\right| \leq|t|$ and glue the obtained annuli, $A^{ \pm}$, identifying points $P \in A^{+}$and $Q \in A^{-}$such that $z^{+}(P) z^{-}(Q)=t$. After this gluing the surfaces $\mathcal{X}^{ \pm}$turn into a single Riemann surface $\mathcal{X}_{t}^{\prime}$ of genus $g^{-}+g^{+}$.

In what follows we derive asymptotical formulas (as $s \rightarrow 0$ ) for basic holomorphic objects (the normalized holomorphic differentials, the canonical meromorphic differential, the prime-form, etc.) on the Riemann surfaces constructed in case I.

The asymptotical formulas (as $t \rightarrow 0$ ) for case $\mathrm{Ib}$ were first derived in 7 . In 27] it was claimed that all the formulas from [7] are incorrect and new ones were proved. Our analysis (in particular, see Example 1 below) shows that formulas from [7. (as well as Fay's proofs of these formulas) are applicable in Case Ia. As was explained to us by Richard Wentworth (private communication), Fay in fact makes a mistake when considering Case Ib: his "pinching parameter" depends in its turn on the deformation parameter and this results in additional terms in asymptotical expansions which were lost in [7. In Case Ia the pinching parameter is independent of the deformation parameter and Fay's scheme works perfectly.

The case of our concern, I, is essentially the same as Case Ia (the pinching parameter, $z$ in equation (2.22) below, is independent of the deformation parameter $s$ ) and we give here the proofs of all the asymptotical formulas for it. Mainly we use the methods similar to those of Fay (where they are applicable), although we have chosen to follow the elementary analytical methods of Yamada (and not those of [7, 17]) when introducing a holomorphic family of Abelian differentials on $\mathcal{X}_{s}$ and studying the analytical properties of the coefficients in the Laurent expansions in the pinching zone. 
2.1. Two examples in genus 0. Canonical meromorphic bidifferential $W$. Recall that to any compact Riemann surface $\mathcal{X}$ of genus $g$ with a chosen canonical basis of cycles $\left\{a_{\alpha}, b_{\alpha}\right\}$ on it one associates the so-called canonical meromorphic bidifferential $W(\cdot, \cdot)$, which

- is a meromorphic one-form with respect to each argument,

- is symmetric, i.e. $W(P, Q)=W(Q, P)$,

- has a single pole at the diagonal $P=Q$ and

$$
W(z(P), z(Q)) \sim \frac{d z(P) d z(Q)}{(z(P)-z(Q))^{2}}+\frac{1}{6} S_{B}(z(Q)) d z(P) d z(Q)+o(1)
$$

as $P \rightarrow Q$, where $S_{B}$ is the Bergman projective connection (see [7]),

- satisfies $\oint_{a_{\alpha}} W(P, \cdot)=0$ for any $P \in \mathcal{X}$ and $\alpha=1, \ldots, g$.

(In case $g=0$ the last condition is void.) The canonical meromorphic bidifferential is related to the prime form via the equation $W(P, Q)=d_{P} d_{Q} \log E(P, Q)$ (see [7]).

Example 1: Case Ia. We start with the following simple statement. Let $\mathcal{X}$ be the two-fold branched covering of the Riemann sphere $\mathbb{P}^{1}$ with branch points $z_{1}$, $z_{2}$. Let $P \in \mathcal{X}$ and $z$ be the projection of $P$ on $\mathbb{P}^{1}$. Then the map

$$
P \mapsto \delta=\sqrt{\frac{z-z_{1}}{z-z_{2}}}
$$

is the biholomorphic isomorphism of $\mathcal{X}$ and $\mathbb{P}^{1}$. Applying to $\delta$ the fractional linear transformation $\delta \mapsto \gamma=\frac{z_{2}-z_{1}}{\delta-1}+z_{2}$, we get the isomorphism

$$
P \mapsto \gamma=z+\sqrt{\left(z-z_{1}\right)\left(z-z_{2}\right)}
$$

of $\mathcal{X}$ and $\mathbb{P}^{1}$ which is more convenient for our future purposes.

Now let $t>0$ and $z_{1}=-\sqrt{t}, z_{2}=\sqrt{t}$. When $t \rightarrow 0$ the Riemann sphere $\mathcal{X}_{t}$ degenerates to the singular Riemann surface with two components, Riemann spheres $S^{+}$and $S^{-}$, attached to each other at the point 0 . So, our situation is exactly the one described in Case Ia.

Let $W_{t}(\cdot, \cdot), W_{-}(\cdot, \cdot)$ and $W_{+}(\cdot, \cdot)$ be the canonical meromorphic bidifferentials on $\mathcal{X}_{t}, S^{-}$and $S^{+}$respectively.

Then the following asymptotics holds:

$$
\begin{aligned}
& W_{t}(z(P), z(Q)) \\
& =\left\{\begin{array}{l}
W_{ \pm}(z(P), z(Q))+\frac{t}{4} W_{ \pm}\left(z(P), 0^{ \pm}\right) W_{ \pm}\left(z(Q), 0^{ \pm}\right)+O\left(t^{2}\right) \quad \text { if } P, Q \in S^{ \pm} \\
-\frac{t}{4} W_{ \pm}\left(z(P), 0^{ \pm}\right) W_{\mp}\left(z(Q), 0^{\mp}\right)+O\left(t^{2}\right) \quad \text { if } P \in S^{ \pm}, Q \in S^{\mp} .
\end{array}\right.
\end{aligned}
$$

(This asymptotics (with the minus sign in the last line lost) was mistakenly stated in ([7], formula (49), p. 41) for two Riemann surfaces glued via plumbing construction (Case Ib); however, being false in Case Ib, it is true in Case Ia.)

Let $P, Q$ be two points of the covering $\mathcal{X}_{t}$ lying on the same sheet (say $S_{+}$) with projections $z$ and $\zeta$; assume for simplicity that $z$ and $\zeta$ are real and positive. 
Using the uniformization map (2.1), one can write the following asymptotics for the canonical meromorphic bidifferential on $\mathcal{X}_{t}$ :

$$
\begin{gathered}
W_{t}(z, \zeta)=\frac{d \gamma(z) d \gamma(\zeta)}{(\gamma(z)-\gamma(\zeta))^{2}}=\frac{\left(1+\frac{z}{\sqrt{z^{2}-t}}\right)\left(1+\frac{\zeta}{\sqrt{\zeta^{2}-t}}\right)}{\left[z-\zeta+\sqrt{z^{2}-t}-\sqrt{\zeta^{2}-t}\right]^{2}} d z d \zeta \\
=\frac{d z d \zeta}{(z-\zeta)^{2}}+\frac{t}{4 z^{2} \zeta^{2}} d z d \zeta+O\left(t^{2}\right) d z d \zeta
\end{gathered}
$$

as $t \rightarrow 0+$, which agrees with Fay's formula (49).

(We remind the reader that the canonical bidifferential $W_{+}$on $S_{+}$(as well as on $\left.S_{-}\right)$is $\frac{d z d \zeta}{(z-\zeta)^{2}}$ and, therefore, $W_{ \pm}\left(z(P), 0^{ \pm}\right) W_{ \pm}\left(z(Q), 0^{ \pm}\right)=\frac{1}{z^{2} \zeta^{2}} d z d \zeta$.)

If $P \in S^{+}, Q \in S^{-}$, then all the " $\zeta$ "- square roots in (2.3) change their sign and we arrive at the second case of Fay's expansion (2.2).

Example 2: Case Ib. This is a rather elementary simplification of Yamada's Example 1 ([27], pp. 140-142). The author thanks D. Korotkin for pointing it out to him.

Let $S^{+}=S_{v}$ and $S^{-}=S_{w}$ be two Riemann spheres with standard coordinates $v$ and $w$ in $S_{v, w} \backslash\{\infty\}$. Also let $\zeta=1 / w$ be the local parameter near the point at infinity of $S_{w}$. Glue $S_{v} \backslash\{|v|<t\}$ and $S_{w} \backslash\{|\zeta|<t\}$ together, identifying the points $v \in\{t \leq|v| \leq 1\} \subset S_{v}$ and $\zeta \in\{t \leq|\zeta| \leq 1\} \subset S_{w}$ such that $v \zeta=v / w=t$. We get a Riemann surface $\mathcal{X}_{t}$ of genus 0 . It is easy to write the uniformization map $\mathcal{X}_{t} \rightarrow S_{z}$, where $S_{z}$ is the Riemann sphere with standard coordinate $z$ in $S_{z} \backslash\{\infty\}$.

Namely, define the map $z: S_{v} \backslash\{|v|<t\} \cup S_{w} \backslash\{|\zeta|<t\} \rightarrow S_{z}$ via $z(v)=v$ for $v \in S_{v} \backslash\{|v|<t\}$ and $z(w)=t w$ for $w \in S_{w} \backslash\{|\zeta|<t\}$. Obviously, the relation $v / w=t$ implies that $z(v)=z(w)$; therefore, the map $z$ gives rise to a biholomorphic $\operatorname{map} \mathcal{X}_{t} \rightarrow S_{z}$.

One has the following obvious relations for the canonical meromorphic bidifferentials $W_{t}, W_{ \pm}$on $\mathcal{X}_{t}$ and $\mathcal{X}_{ \pm}$:

$$
W_{t}\left(z_{1}, z_{2}\right)=\frac{d z_{1} d z_{2}}{\left(z_{1}-z_{2}\right)^{2}}=\frac{d v_{1} d v_{2}}{\left(v_{1}-v_{2}\right)^{2}}=W_{+}\left(v_{1}, v_{2}\right)
$$

if $v_{1}, v_{2} \in S^{+} \backslash\{|v| \leq 1\}$;

$$
W_{t}\left(z_{1}, z_{2}\right)=\frac{d z_{1} d z_{2}}{\left(z_{1}-z_{2}\right)^{2}}=\frac{d\left(t w_{1}\right) d\left(t w_{2}\right)}{\left(t w_{1}-t w_{2}\right)^{2}}=\frac{d w_{1} d w_{2}}{\left(w_{1}-w_{2}\right)^{2}}=W_{-}\left(w_{1}, w_{2}\right)
$$

if $w_{1}, w_{2} \in S^{-} \backslash\{|\zeta| \leq 1\}$;

$$
W_{t}\left(z_{1}, z_{2}\right)=\frac{d z_{1} d z_{2}}{\left(z_{1}-z_{2}\right)^{2}}=\frac{d v d(t w)}{(v-t w)^{2}}=t \frac{d v d w}{v^{2}}+O\left(t^{2}\right)
$$

as $t \rightarrow 0$ if $v \in S^{+} \backslash\{|w| \leq 1\}$ and $w \in S^{-} \backslash\{|\zeta| \leq 1\}$ in complete agreement with Yamada's asymptotical formulas for Case Ib:

$$
W_{t}\left(z_{1}, z_{2}\right)=\left\{\begin{array}{r}
W_{+}\left(v_{1}, v_{2}\right)+\left.t^{2} S_{B}(\zeta)\right|_{\zeta=0} W_{+}\left(v_{1}, 0\right) W_{+}\left(v_{2}, 0\right)+O\left(t^{3}\right) \\
\text { for } v_{1}, v_{2} \in S^{+} \backslash\{|v| \leq 1\} \\
W_{-}\left(w_{1}, w_{2}\right)+\left.t^{2} S_{B}(v)\right|_{v=0} W_{-}\left(w_{1}, \infty\right) W_{-}\left(w_{2}, \infty\right)+O\left(t^{3}\right) \\
\text { for } w_{1}, w_{2} \in S^{-} \backslash\{|\zeta| \leq 1\} \\
-t W_{+}(v, 0) W_{-}(w, \infty)+O\left(t^{2}\right) \\
\text { for } v \in S^{+} \backslash\{|v| \leq 1\}, w \in S^{-} \backslash\{|\zeta| \leq 1\}
\end{array}\right.
$$


(see [27, formula (15) on p. 122; it should be noted that for coordinates $v$ and $\zeta$ on the Riemann sphere one has $\left.S_{B}(v)=S_{B}(\zeta) \equiv 0\right)$.

2.2. Asymptotical formulas. Here we deal with Case I, assuming that the genera of the surfaces $\mathcal{X}^{ \pm}$are greater than zero.

Denote the part of the Riemann surface $\mathcal{X}_{s}$ which came from the discs $D^{ \pm}$after the gluing procedure by $\mathcal{U}$. The domain $\mathcal{U}$ is an open (topological) annulus and the map $z$ can be considered as defined on $\mathcal{U}$. The map

$$
z: \mathcal{U} \rightarrow\{z \in \mathbb{C}:|z|<1\}
$$

defines a two-sheeted covering of the disc $\{|z|<1\}$ ramified over $z=0$ and $z=s$, whereas the map

$$
\mathcal{U} \ni P \mapsto X=z-\frac{s}{2}+\sqrt{z(z-s)}
$$

is a well-defined biholomorphic bijection (of course, the value of the square root depends on which disk, $D^{+}$or $D^{-}$, the point $P$ belongs; one also has to fix a branch of the square root, say, for the disk $D^{+}$with the cut between 0 and $s$; there are two choices and we make one once and forever).

(It should be noted that map (2.9) (being appropriately extended) uniformizes the two-sheeted covering of the Riemann sphere branched over the points 0 and $s$. The image of the point at infinity of the first sheet is $\infty$; the image of the point at infinity of the second one is 0 .)

For sufficiently small $s$ the annulus

$$
\mathbb{A}_{s}=\left\{P: \frac{|s|^{2}}{4}<|X|<1\right\}
$$

belongs to $\mathcal{U}$. Moreover, the boundary curve $|X|=1$ lies in a small vicinity of the circle $|z|=1 / 2$ of the "+"-sheet of the covering (2.8), whereas the boundary curve $|X|=|s|^{2} / 4$ lies in a small vicinity of the circle $|z|=1 / 2$ of the "-"-sheet.

The following two lemmas are analogs of Yamada's Theorem 1 and Lemma 1 (27], p. 116) for the family $\mathcal{X}_{s}$. We follow the proofs of Yamada, making necessary (in fact, rather minor) modifications.

Lemma 1. Let $v_{ \pm}$be holomorphic differentials on $\mathcal{X}^{ \pm}$. There exists a holomorphic differential $w_{s}$ on $\mathcal{X}_{s}$ such that for any $\rho, \sqrt{|s|}<\rho<1$, the inequality

$$
\left\|w_{s}-v_{+}\right\|_{\Omega_{\rho}^{+}}+\left\|w_{s}-v_{-}\right\|_{\Omega_{\rho}^{-}} \leq C(\rho)|s|
$$

holds, where

$$
\begin{gathered}
\Omega_{\rho}^{+}=\mathcal{X}^{+} \backslash\left\{P \in D^{+}:|X(P)| \leq \rho\right\}, \\
\Omega_{\rho}^{-}=\mathcal{X}^{-} \backslash\left\{P \in D^{-}:|X(P)| \geq|s|^{2} /(4 \rho)\right\} .
\end{gathered}
$$

Here, as usual, the $L_{2}$-norm of a one-form in a subdomain $\Omega$ of a Riemann surface is defined via

$$
\|u\|_{\Omega}=\iint_{\Omega} u \wedge \overline{* u}
$$

Remark. The curves $|X|=\sqrt{|s|}$ and $|X|=|s|^{3 / 2} / 4$ belong to small vicinities of the circles $|z|=\sqrt{|s|} / 2$ lying on the "+" and "-" sheets of the covering (2.8) respectively. 
Proof. Let $\int_{0}^{z} u_{+}=\sum_{n=1}^{\infty} \alpha_{n} z^{n}$ near $P^{+}$; after passing to coordinate $X$,

$$
z=\frac{X}{2}+\frac{s}{2}+\frac{s^{2}}{8 X}
$$

we get

$$
f_{+}(z)=\int_{0}^{z} u^{+}=\sum_{n=1}^{\infty} a_{n}^{+}(s) X^{n}+a_{0}(s)+\sum_{n=-\infty}^{-1} a_{n}^{-}(s) X^{n}
$$

where

$$
a_{n}^{+}(s)=\alpha_{n}\left(1 / 2^{n}+O(s)\right), \quad a_{0}(s)=O(s), \quad a_{n}^{-}(s)=O\left(s^{2|n|}\right),
$$

as $s \rightarrow 0$.

Analogously, from the expansion $f_{-}(z)=\int_{0}^{z} u_{-}=\sum_{n=1}^{\infty} \beta_{n} z^{n}$ near $P^{-}$one gets

$$
\int_{0}^{z} u_{-}=\sum_{n=1}^{\infty} b_{n}^{+}(s) X^{n}+b_{0}(s)+\sum_{n=-\infty}^{-1} b_{n}^{-}(s) X^{n}
$$

where

$$
b_{n}^{+}(s)=\beta_{n}\left(1 / 2^{n}+O(s)\right), \quad b_{0}(s)=O(s), \quad b_{n}^{-}(s)=O\left(s^{2|n|}\right),
$$

as $s \rightarrow 0$.

Now, 27, we are to construct a sequence, $\Phi_{s}^{(k)}$, of $C^{1}$-forms on $\mathcal{X}_{s}$ coinciding with $v_{ \pm}$in $\Omega_{\rho}^{ \pm}$and such that

$$
\left\|\Phi_{s}^{(k)}-i * \Phi_{s}^{(k)}\right\|^{2} \leq O\left(s^{2}\right)+1 / k .
$$

For a harmonic function $h_{s}$ in the annulus $\left\{|s|^{2} /(4 \rho) \leq|X| \leq \rho\right\}$ with boundary values $f_{-}$and $f_{+}$one has the relation

$$
\begin{gathered}
\quad \frac{1}{2 \pi} \iint_{|s|^{2} /(4 \rho) \leq|X| \leq \rho}\left(\left|\partial_{X} h_{s}\right|^{2}+\left|\partial_{\bar{X}} h_{s}\right|^{2}\right) \frac{|d X \wedge \overline{d X}|}{2} \\
=\sum_{n=1}^{\infty} \frac{n\left|b_{-n}^{-}-a_{-n}^{-}\right|^{2}}{\rho^{2 n}-\left(\frac{|s|^{2}}{4 \rho}\right)^{2 n}}+\sum_{n=1}^{\infty} \frac{n\left|b_{n}^{+}-a_{n}^{+}\right|^{2}}{\rho^{-2 n}-\left(\frac{|s|^{2}}{4 \rho}\right)^{-2 n}}+\frac{\left|b_{0}-a_{0}\right|^{2}}{2 \log \left(\frac{\rho^{2}}{|s|^{2} / 4}\right)}=O\left(s^{2}\right) .
\end{gathered}
$$

It can be shown (say, via polynomial interpolation along radial directions) that one can change the function $h_{s}$ in small vicinities of the boundary circles $|X|=\rho$ and $|X|=\left|s^{2}\right| /(4 \rho)$ obtaining the function $h_{s}^{(k)}$ such that

$$
\iint_{|s|^{2} /(4 \rho) \leq|X| \leq \rho}\left(\left|\partial_{X}\left(h_{s}-h_{s}^{(k)}\right)\right|^{2}+\left|\partial_{\bar{X}}\left(h_{s}-h_{s}^{(k)}\right)\right|^{2}\right) \frac{|d X \wedge \overline{d X}|}{2} \leq \frac{1}{k}
$$

and the 1 -form

$$
\Phi_{s}^{(k)}=\left\{\begin{array}{l}
v_{ \pm} \text {in } \Omega_{\rho}^{ \pm}, \\
d\left(h_{s}^{(k)}\right) \text { in } \mathcal{X}_{s} \backslash\left(\Omega_{\rho}^{+} \cup \Omega_{\rho}^{-}\right)
\end{array}\right.
$$

is $C^{1}$-smooth. Since the operator Id $-i *$ kills the $(1,0)$-forms, the inequality (2.11) follows from (2.12) and (2.13).

Decomposing $(\mathrm{Id}-i *) \Phi_{s}^{(k)}$ into the $\left(L_{2}\right.$-orthogonal!) sum of a harmonic one-form $\omega_{h}$, an exact form $\omega_{e}$ and a co-exact form $\omega_{e}^{*}$ (see [1], Chapter V; here "exact form" means a form belonging to the $L_{2}$-closure of the space of smooth exact forms), we observe that the left part of the equation

$$
\Phi_{s}^{(k)}-\omega_{e}=i * \Phi_{s}^{(k)}+\omega_{h}+\omega_{e}^{*}
$$


is a closed form, whereas its left part is co-closed; therefore, both are harmonic by virtue of Weyl's Lemma (see [1, Chapter V).

Now, applying to the harmonic form $\Phi_{s}^{(k)}-\omega_{e}$ the operator $\frac{1}{2}(\operatorname{Id}+i *)$ one gets a holomorphic one-form

$$
\Psi_{s}^{(k)}=\frac{1}{2}(\mathrm{Id}+i *)\left[\Phi_{s}^{(k)}-\omega_{e}\right]
$$

which coincides with $v_{ \pm}+\frac{1}{2}(\mathrm{Id}+i *) \omega_{e}$ in $\Omega_{\rho}^{ \pm}$. Therefore,

$$
\left\|\Psi_{s}^{(k)}-v_{+}\right\|_{\Omega_{\rho}^{+}}^{2}+\left\|\Psi_{s}^{(k)}-v_{-}\right\|_{\Omega_{\rho}^{-}}^{2} \leq \frac{1}{4}\left\|\omega_{e}+i * \omega_{e}\right\| \leq \frac{1}{2}|| \omega_{e}\left\|\leq \frac{1}{2}\right\| \Phi_{s}^{(k)}-i * \Phi_{s}^{(k)} \|
$$

and

$$
\left\|\Psi_{s}^{(k)}-v_{+}\right\|_{\Omega_{\rho}^{+}}^{2}+\left\|\Psi_{s}^{(k)}-v_{-}\right\|_{\Omega_{\rho}^{-}}^{2} \leq O\left(s^{2}\right)+\frac{1}{k}
$$

by virtue of (2.11).

Choosing from the sequence $\left\{\Psi_{s}^{(k)}\right\}_{k \geq 1}$ a converging subsequence (uniform $L_{2^{-}}$ boundedness of holomorphic forms on a compact Riemann surface implies uniform boundedness of their coefficients) and passing to the limit $k \rightarrow \infty$, we get a holomorphic 1-form $w_{s}$ with all the needed properties.

Remark 2. Actually a stronger variant of Lemma 1 is true: the differentials $v_{ \pm}$can be meromorphic with poles lying outside of $D^{ \pm}$. In this case the differential $w_{s}$ is also meromorphic and has the same singularities as $v_{ \pm}$.

Now choose on $\mathcal{X}^{ \pm}$a canonical basis of cycles $\left\{a_{\alpha}^{ \pm}, b_{\alpha}^{ \pm}\right\}_{\alpha=1, \ldots, g^{ \pm}}$such that none of the cycles intersects the disk $D^{ \pm}$. Also let $\left\{u_{\alpha}^{ \pm}\right\}_{\alpha=1, \ldots, g^{ \pm}}$be the corresponding basis of normalized differentials.

The set of cycles

$$
\left\{a_{\alpha}, \beta_{\alpha}\right\}_{\alpha=1, \ldots, g^{+}+g^{-}}=\left\{a_{1}^{+}, \ldots, a_{g^{+}}^{+}, a_{1}^{-}, \ldots, a_{g^{-}}^{-} ; b_{1}^{+}, \ldots, b_{g^{+}}^{+}, b_{1}^{-}, \ldots, b_{g^{-}}^{-}\right\}
$$

forms a canonical basis on the Riemann surface $\mathcal{X}_{s}$. Let $\left\{v_{\alpha}^{(s)}\right\}_{\alpha=1, \ldots, g^{-}+g^{+}}$be the corresponding basis of normalized holomorphic differentials on $\mathcal{X}_{s}$.

Also let $w_{\alpha}^{(s)}$ be a holomorphic one-form on $\mathcal{X}_{s}$ which is constructed in Lemma 1 when one takes $\left(v_{+}, v_{-}\right)=\left(v_{\alpha}^{+}, 0\right)$ for $\alpha=1, \ldots, g^{+}$and $\left(v_{+}, v_{-}\right)=\left(0, v_{\alpha-g^{+}}^{-}\right)$for $\alpha=g^{+}+1, \ldots, g^{+}+g^{-}$.

The corresponding matrix of $a$-periods $\mathbb{P}=\left\|\oint_{a_{\alpha}} w_{\beta}^{(s)}\right\|_{\alpha, \beta=1, \ldots, g^{+}+g^{-}}$satisfies

$$
\mathbb{P}=I_{g^{+}+g^{-}}+O(s)
$$

as $s \rightarrow 0$ due to Lemma 1 . This immediately implies the following lemma.

Lemma 2. The basis $\left\{v_{\alpha}^{(s)}\right\}_{\alpha=1, \ldots, g^{+}+g^{-}}$of normalized holomorphic differentials on $\mathcal{X}_{s}$ satisfies

$$
\left(v_{1}^{(s)}, \ldots, v_{g^{+}+g^{-}}^{(s)}\right)=\left(I_{g^{-}+g^{+}}+O(s)\right)\left(w_{1}^{(s)}, \ldots, w_{g^{+}+g^{-}}^{(s)}\right) ;
$$

in particular, all the differentials $v_{\alpha}^{(s)}$ are uniformly (with respect to $s$ ) bounded in, say, $\mathcal{X}_{s} \backslash\left\{P \in \mathcal{X}_{s},|z(P)|<1 / 4\right\}$. 
Laurent expansion for basic holomorphic differentials. Writing the differential $v_{\alpha}^{(s)}$ as $v_{\alpha}^{(s)}(X) d X$ in the local parameter $X=z-\frac{s}{2}+\sqrt{z(z-s)}$ and expanding the coefficient $v_{\alpha}^{(s)}(\cdot)$ in the Laurent series in the annulus $|s|^{2} / 4<|X|<1$, one gets

$$
v_{\alpha}^{(s)}(X) d X=\left(\sum_{n>o} \gamma_{-n}(s) X^{-n}+\sum_{n \geq 0} \gamma_{n}(s) X^{n}\right) d X .
$$

Observe that $d X=\frac{X d z}{\sqrt{z(z-s)}}$ and for $n \geq 0$ one has

$$
\begin{aligned}
X^{n} d X & =\frac{(z-s / 2+\sqrt{z(z-s)})^{n+1}}{\sqrt{z(z-s)}} d z \\
& =\left\{\sum_{k=0}^{n+1} p_{k}(s) z^{k}+\frac{1}{\sqrt{(z(z-s)}} \sum_{k=0}^{n+1} q_{k}(s) z^{k}\right\} d z
\end{aligned}
$$

with some polynomials $p_{k}(s), q_{k}(s)$. On the other hand, since

$$
(z-s / 2+\sqrt{z(z-s)})(z-s / 2-\sqrt{z(z-s)})=s^{2} / 4,
$$

for $n>0$ one has

$$
\begin{array}{r}
X^{-n} d X=\frac{4^{n}}{s^{2 n}} \frac{(z-s / 2-\sqrt{z(z-s)})^{n}(z-s / 2+\sqrt{z(z-s)})}{\sqrt{z(z-s)}} d z \\
=\frac{1}{s^{2 n-2}}\left\{\sum_{k=0}^{n-1} \tilde{p}_{k}(s) z^{k}+\frac{1}{\sqrt{z(z-s)}} \sum_{k=0}^{n-1} \tilde{q}_{k}(s) z^{k}\right\} d z
\end{array}
$$

with some polynomials $\tilde{p}_{k}(s), \tilde{q}_{k}(s)$.

For $n>0$ one has

$$
\begin{aligned}
\gamma_{-n}(s) & =\frac{1}{2 \pi i} \int_{|X|=|s|^{2} / 4} v_{\alpha}^{(s)}(X) X^{n-1} d X \\
& =\frac{1}{2 \pi i} \int_{\Gamma_{-}} v_{\alpha}^{(s)}(z)(z-s / 2+\sqrt{z(z-s)})^{n-1} d z \\
& =\int_{\Gamma_{-}} O(1) \times O\left(s^{2 n-2}\right) d z=O\left(s^{2 n-2}\right)
\end{aligned}
$$

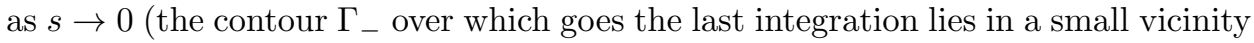
of the circle $|z|=1 / 2$ of the "-"-sheet; the factor $v_{\alpha}^{(s)}(z)$ is uniformly bounded on this contour with respect to $s$ by virtue of Lemma 2).

In the same manner for $n \geq 0$ one has

$$
\gamma_{n}(s)=\frac{1}{2 \pi i} \int_{|X|=1} \frac{v_{\alpha}^{(s)}(X)}{X^{n+1}} d X=\frac{1}{2 \pi i} \int_{\Gamma_{+}} \frac{v_{\alpha}^{(s)}(z) d z}{(z-s / 2+\sqrt{z(z-s)})^{n+1}}=O(1) .
$$

(The contour $\Gamma_{+}$lies in a small vicinity of the circle $|z|=1 / 2$ of the "+"-sheet, the factor $v_{\alpha}^{(s)}(z)$ is uniformly bounded by virtue of Lemma 2 , and the denominator of the integrand is close to 1.) 
Now from (2.17), (2.18) and (2.19) together with the estimates (2.21) and (2.20) one gets the expansion

$$
v_{\alpha}^{(s)}(z) d z=\sum_{k=0}^{\infty} a_{k}(s) z^{k} d z+\frac{1}{\sqrt{z(z-s)}} \sum_{k=o}^{\infty} b_{k}(s) z^{k} d z,
$$

where the coefficients $a_{k}, b_{k}$ are analytic near $s=0$. This expansion is valid in the zone $\left\{|s|^{2} / 4<|X|<1\right\}$ (the latter for small $s$ is close to the set $\left\{P \in \mathcal{X}_{s}:|z(P)| \leq\right.$ $1 / 2\})$.

Remark 3. Expansion (2.22) is a complete analog of Fay's expansion stated on page 40 of [7] for the deformation family in Case Ib. However, it is important here that in (2.22) the parameter $z$ is $s$-independent whereas in the expansion from [7] the pinching parameter $\chi$ depends on the deformation parameter. The latter fact was missed in [7] (in particular, the last formula on page 40 of [7] should contain more terms on the right-hand side) (23]).

Main asymptotical formulas for basic holomorphic differentials and the canonical meromorphic bidifferential. Let $W, W_{ \pm}$be the canonical meromorphic bidifferentials on $\mathcal{X}_{s}$ and $\mathcal{X}^{ \pm}$respectively.

Theorem 1. For $\alpha=1, \ldots, g^{+}$, one has the asymptotics as $s \rightarrow 0$,

$$
v_{\alpha}^{(s)}(P)=\left\{\begin{array}{l}
u_{\alpha}^{+}(P)+\frac{s^{2}}{16} u_{\alpha}^{+}\left(P_{+}\right) W_{+}\left(P, P_{+}\right)+o\left(s^{2}\right) \quad \text { if } P \in L^{+} \backslash D^{+} \subset \mathcal{X}_{s} \\
-\frac{s^{2}}{16} u_{\alpha}^{+}\left(P_{+}\right) W_{-}\left(P, P_{-}\right)+o\left(s^{2}\right) \quad \text { if } P \in \mathcal{X}^{-} \backslash D^{-} \subset \mathcal{X}_{s} .
\end{array}\right.
$$

For $\alpha=g^{+}+k, k=1, \ldots, g^{-}$, one has

$$
v_{\alpha}^{(s)}(P)=\left\{\begin{array}{l}
u_{k}^{-}(P)+\frac{s^{2}}{16} u_{k}^{-}\left(P_{-}\right) W_{-}\left(P, P_{-}\right)+o\left(s^{2}\right) \quad \text { if } P \in L^{-} \backslash D^{-} \subset \mathcal{X}_{s} \\
-\frac{s^{2}}{16} u_{k}^{-}\left(P_{-}\right) W_{+}\left(P, P_{+}\right)+o\left(s^{2}\right) \quad \text { if } P \in \mathcal{X}^{+} \backslash D^{+} \subset \mathcal{X}_{s} .
\end{array}\right.
$$

Here the values of the differentials at the points $P_{ \pm}$are calculated in the local parameter $z$, and the values of the differentials at $P \in \mathcal{X}^{ \pm} \backslash D^{ \pm} \subset \mathcal{X}_{s}$ are calculated in an arbitrary local parameter inherited from $\mathcal{X}^{ \pm}$(of course, the same for the $l$. h. s. and the r. h. s.).

Theorem 2. For the canonical meromorphic bidifferential on $\mathcal{X}_{s}$ one has the following asymptotics as $s \rightarrow 0$ :

$$
W(R, S)=\left\{\begin{array}{l}
W_{+}(R, S)+\frac{s^{2}}{16} W_{+}\left(R, P_{+}\right) W_{+}\left(S, P_{+}\right) \text {if } R, S \in \mathcal{X}^{+} \backslash D^{+} \subset \mathcal{X}_{s}, \\
-\frac{s^{2}}{16} W_{+}\left(R, P_{+}\right) W_{-}\left(S, P_{-}\right) \text {if } R \in \mathcal{X}^{+} \backslash D^{+} \subset \mathcal{X}_{s} ; S \in \mathcal{X}^{-} \backslash D^{-} \subset \mathcal{X}_{s}, \\
W_{-}(R, S)+\frac{s^{2}}{16} W_{-}\left(R, P_{-}\right) W_{-}\left(S, P_{-}\right) \text {if } R, S \in \mathcal{X}^{-} \backslash D^{-} \subset \mathcal{X}_{s} .
\end{array}\right.
$$

Proof. Observe that $\lim _{s \rightarrow 0} \sqrt{z(P)(z(P)-s)}= \pm z(P)$ if $P \in D^{ \pm} \backslash[0, s] \subset \mathcal{X}_{s}$. Let $\alpha=1, \ldots, g^{+}$. Taking two points in $\mathcal{U}$ with $z(P)=z$ and sending $s \rightarrow 0$ in (2.22), one gets

$$
u_{\alpha}^{+}(z) d z=\left(\sum_{k=0}^{\infty} a_{k}(0) z^{k}+\sum_{k=0}^{\infty} b_{k}(0) z^{k-1}\right) d z
$$

for the point on the "+"-sheet and

$$
0=\sum_{k=0}^{\infty} a_{k}(0) z^{k}-\sum_{k=o}^{\infty} b_{k}(0) z^{k-1}
$$


for the point on the "-"-sheet. This implies the relations

$$
b_{0}(0)=0
$$

and

$$
\frac{u_{\alpha}^{+}\left(P_{+}\right)}{2}=a_{0}(0)=b_{1}(0)
$$

For $P \in D^{+}$one has

$$
\begin{aligned}
\frac{1}{s}\left(v_{\alpha}^{(s)}-v_{\alpha}^{(0)}\right) & =\sum_{k \geq 0}^{\infty} \frac{a_{k}(s)-a_{k}(0)}{s} z^{k} d z \\
& =\sum_{k \geq 0}\left\{\frac{b_{k}(s)-b_{k}(0)}{s} \frac{z^{k}}{\sqrt{z(z-s)}}+b_{k}(0) z^{k-1} \frac{\frac{z}{\sqrt{z(z-s)}}-1}{s}\right\} d z \\
& =\left\{\sum_{k=0}^{\infty} a_{k}^{\prime}(0) z^{k}+\sum_{k=0}^{\infty} b_{k}^{\prime}(0) z^{k-1}+\frac{1}{2} \sum_{k=0}^{\infty} b_{k}(0) z^{k-2}+O(s)\right\} d z .
\end{aligned}
$$

Since $b_{0}(0)=0$, the limit of the left-hand side of (2.28) as $s \rightarrow 0$ is a meromorphic differential on $\mathcal{X}^{+}$with a single pole at $P_{+}$; therefore, it is a holomorphic differential, i.e.

$$
b_{0}^{\prime}(0)+\frac{1}{2} b_{1}(0)=0 .
$$

Moreover, since all the $a$-periods of this differential vanish, it equals zero.

Then, again for a point on the "+"-sheet, we have

$$
\begin{aligned}
& \frac{1}{s^{2}}\left(v_{\alpha}^{(s)}-v_{\alpha}^{(0)}\right)=\frac{1}{s^{2}} {\left[\sum_{k \geq 0}\left(a_{k}(0)+s a_{k}^{\prime}(0)+\frac{s^{2}}{2} a_{k}^{\prime \prime}(0)+O\left(s^{3}\right)\right) z^{k}\right.} \\
&+\sum_{k \geq 0}\left(b_{k}(0)+s b_{k}^{\prime}(0)+\frac{s^{2}}{2} b_{k}^{\prime \prime}(0)+O\left(s^{3}\right)\right) z^{k-1}\left(1+\frac{s}{2 z}+\frac{3}{8} \frac{s^{2}}{z^{2}}+O\left(s^{3}\right)\right) \\
& \\
&\left.-\sum_{k \geq 0} a_{k}(0) z^{k}-\sum_{k \geq 0} b_{k}(0) z^{k-1}\right] d z
\end{aligned}
$$

Since the $s$-linear term in the braces vanishes, the limit of this expression as $s \rightarrow 0$ equals

$$
\left[\sum_{k=0}^{\infty} \frac{a_{k}^{\prime \prime}(0)}{2} z^{k}+\frac{b_{k}^{\prime \prime}(0)}{2} z^{k-1}+\frac{3}{8} b_{k}(0) z^{k-3}+\frac{b_{k}^{\prime}(0)}{2} z^{k-2}\right] d z .
$$

Thus the limit is a meromorphic differential on $\mathcal{X}^{+}$with a single pole of the second order $\left(b_{0}(0)=0\right.$ !); the corresponding Laurent coefficient is

$$
\frac{3}{8} b_{1}(0)+\frac{b_{0}^{\prime}(0)}{2}=\frac{b_{1}(0)}{8}=\frac{1}{16} u_{\alpha}^{+}\left(P_{+}\right)
$$

due to (2.27) and (2.29). All the $a$-periods of this differential vanish; therefore, it coincides with

$$
\frac{1}{16} u_{\alpha}^{+}\left(P_{+}\right) W_{+}\left(\cdot, P_{+}\right)
$$

and the first asymptotics in (2.23) is proved. 
The other asymptotics of Theorem 1 can be proved in a similar way. Theorem 2 follows from Theorem 1 (see 7], p. 41 for a short explanation of this implication).

It is also possible to prove Theorem 2 independently. One starts from the generalization of Lemma 1 given in Remark 2 Using this generalization with, say, $v_{-}=0$ and $v_{+}=W_{+}(\cdot, Q)$ with $Q \in \mathcal{X}^{+} \backslash D^{+}$, one establishes expansion (2.22) for the one-form $W(\cdot, Q)$ exactly in the same manner as was done for a basic holomorphic differential. Repeating the proof of Theorem 1 with $W(\cdot, Q)$ instead of $v_{\alpha}^{(s)}$ we arrive at the asymtotics stated in Theorem 2 .

The following proposition gives the asymptotics of a type other than that given in Theorem 2 now one of the arguments of the canonical meromorphic bidifferential lies inside the pinching zone (being one of the two endpoints of the cut).

Proposition 1. Let a point $P$ lie on the surface $\mathcal{X}^{ \pm}$far from the pinching zone and let $P_{r}=z^{-1}(s)$ and $P_{l}=z^{-1}(0)$ be the critical points of the map $z: \mathcal{U} \rightarrow\{z:$ $|z|<1\}$. Then

$$
\begin{gathered}
W\left(P, P_{r}\right)=\frac{\sqrt{s}}{2} W_{ \pm}\left(P_{ \pm}, P\right)+O\left(s^{3 / 2}\right), \\
W\left(P, P_{l}\right)=-i \frac{\sqrt{s}}{2} W_{ \pm}\left(P_{ \pm}, P\right)+O\left(s^{3 / 2}\right),
\end{gathered}
$$

as $s \rightarrow 0$. Here the differentials are calculated in the local parameters related to the corresponding branched coverings: i.e., $\sqrt{z(\cdot)-s}$ at $P_{r}, \sqrt{z(\cdot)}$ at $P_{l}, z^{ \pm}(\cdot)$ at $P_{ \pm}$ and an arbitrary local parameter inherited from $\mathcal{X}^{ \pm}$at $P$.

Proof. For the 1-form $W(\cdot, P)$ one has the expansion (2.22) with $b_{0}(0)=0, b_{0}^{\prime}(0)+$ $\frac{1}{2} b_{1}(0)=0$ and $b_{1}(0)=a_{0}(0)=\frac{1}{2} W_{ \pm}\left(P_{ \pm}, P\right)$.

Now substituting in this expansion $z=s+t^{2}, d z=2 t d t$, setting $t=0$ and then sending $s \rightarrow 0$, we get (2.30). Substituting $z=t^{2}, d z=2 t d t$, setting $t=0$ and sending $s \rightarrow 0$, we get (2.31).

2.3. Asymptotics of $E(P, Q), \sigma(P, Q), C(P)$ and $K^{P}$. First recall the following expression, relating the prime form, $E(x, y)$, to the canonical meromorphic bidifferential on an arbitrary compact Riemann surface of genus $g$ (see [7, p. 26):

$$
\frac{\theta\left(\int_{x}^{y} \vec{v}-e\right) \theta\left(\int_{x}^{y} \vec{v}+e\right)}{\theta^{2}(e) E^{2}(x, y)}=W(x, y)+\sum_{i, j=1}^{g} \frac{\partial^{2} \log \theta(e)}{\partial z_{i} \partial z_{j}} v_{i}(x) v_{j}(y),
$$

where $\vec{v}=\left(v_{1}, \ldots, v_{g}\right)^{t}$ is a column of basic holomorphic differentials, and $e$ is an arbitrary vector from $\mathbb{C}^{n}$.

From this expression, taken together with the asymptotics for the basic holomorphic differentials and the canonical meromorphic bidifferential, one easily derives the following asymptotics for the prime form on the family $\mathcal{X}_{s}$ :

$$
E^{2}(P, Q)=E_{ \pm}^{2}(P, Q)+o(1)
$$

as $s \rightarrow 0$; here the points $P, Q$ belong to $\mathcal{X}^{ \pm}$and are far from the pinching zone, $E_{ \pm}(P, Q)$ is the prime form on $\mathcal{X}^{ \pm}$, and all the prime forms are calculated in local parameters near $P$ and $Q$ inherited from $\mathcal{X}^{ \pm}$; 


$$
E^{2}(P, Q)=-\frac{16}{s^{2}} E_{ \pm}^{2}\left(P, P_{ \pm}\right) E_{\mp}^{2}\left(Q, P_{\mp}\right)+O\left(\frac{1}{s}\right)
$$

if $P \in \mathcal{X}^{ \pm}$and $Q \in \mathcal{X}^{\mp}$;

$$
E^{2}\left(P, P_{r}\right)=\frac{2}{\sqrt{s}} E_{ \pm}^{2}\left(P, P_{ \pm}\right)+O(\sqrt{s}), \quad E^{2}\left(P, P_{l}\right)=\frac{2 i}{\sqrt{s}} E_{ \pm}^{2}\left(P, P_{ \pm}\right)+O(\sqrt{s})
$$

if $P \in \mathcal{X}^{ \pm}$, the local parameter at $P_{l}$ is $\sqrt{z}$, and the local parameter near $P_{r}$ is $\sqrt{z-s}$.

From now on we use the following notation, $\Delta(s)=\frac{4}{s}$, and denote by a single letter $\epsilon$ different unitary constants ("phase factors", $|\epsilon|=1$ ) which may appear as additional factors in some of our formulas; the concrete values of these factors are of no interest for us.

The next two quantities whose asymptotics we need are defined as follows (see [8], (1.13) and (1.17)):

$$
\sigma(P, Q)=\exp \left\{-\sum_{k=1}^{g} \int_{a_{k}} v_{k}(x) \log \frac{E(x, P)}{E(x, Q)}\right\}
$$

and

$$
C(P)=\frac{\theta\left(\int_{P}^{Q_{1}} \vec{v}+\cdots+\int_{P}^{Q_{g}} \vec{v}+K_{P}\right) \prod_{i<j}^{g} E\left(Q_{i}, Q_{j}\right) \prod_{i=1}^{g} \sigma\left(Q_{i}, P\right)}{\operatorname{det}\left(v_{i}\left(Q_{j}\right)\right) \prod_{i=1}^{g} E\left(P, Q_{i}\right)}
$$

where $Q_{1}, \ldots, Q_{g}$ are arbitrary points of $\mathcal{X}$ (expression (2.37) is independent of the choice of these points) and $K_{P}$ is the vector of Riemann constants.

Using asymptotics for the prime-form (2.33 2.35) and the basic holomorphic differentials, one easily obtains from (2.36) the following asymptotics as $s \rightarrow 0$ :

$$
\sigma(P, Q) \sim \sigma_{ \pm}(P, Q)\left[\frac{E_{ \pm}\left(Q, P_{ \pm}\right)}{E_{ \pm}\left(P, P_{ \pm}\right)}\right]^{g^{\mp}}
$$

for $P, Q \in \mathcal{X}^{ \pm}$

$$
\sigma(P, Q) \sim \epsilon \sigma_{ \pm}\left(P, P_{ \pm}\right) \sigma_{\mp}\left(P_{\mp}, Q\right) \frac{\left[E_{\mp}\left(P_{\mp}, Q\right)\right]^{g^{ \pm}}}{\left[E_{ \pm}\left(P, P_{ \pm}\right)\right]^{g^{\mp}}}[\Delta(s)]^{g^{ \pm}-g^{\mp}},
$$

if $P \in \mathcal{X}^{ \pm}, Q \in \mathcal{X}^{\mp}$

$$
\sigma\left(P_{r}, Q\right) \sim \epsilon \sigma\left(P_{l}, Q\right) \sim \epsilon \sigma_{ \pm}\left(P_{ \pm}, Q\right)\left[E_{ \pm}\left(P_{ \pm}, Q\right)\right]^{g^{\mp}}[\Delta(s)]^{\left(3 g^{\mp}-g^{ \pm}\right) / 4}
$$

if $Q \in \mathcal{X}^{ \pm}$.

The asymptotics of (2.37) is a bit more tricky to obtain and we give more details. First choose the points $\left\{Q_{i}\right\}$ in such a way that $g^{+}$of them, $R_{1}, \ldots, R_{g^{+}}$belong to $\mathcal{X}^{+}$and the other $g^{-}$points, $S_{1}, \ldots, S_{g^{-}}$, belong to $\mathcal{X}^{-}$. Then, assuming for 
definiteness $P \in \mathcal{X}^{+}$, one has as $s \rightarrow 0$,

$$
\begin{aligned}
\theta\left(\int_{P}^{Q_{1}} \vec{v}+\cdots+\int_{P}^{Q_{g}} \vec{v}+K_{P} \mid \mathbb{B}\right) \\
\sim \theta\left(\int_{P}^{R_{1}}\left(\begin{array}{l}
\vec{v}_{+} \\
\overrightarrow{0}
\end{array}\right)+\cdots+\int_{P}^{R_{g^{+}}}\left(\begin{array}{l}
\vec{v}_{+} \\
\overrightarrow{0}
\end{array}\right)+g_{-} \int_{P}^{P_{+}}\left(\begin{array}{l}
\vec{v}_{+} \\
0
\end{array}\right)+\int_{P_{-}}^{S_{1}}\left(\begin{array}{l}
\overrightarrow{0}_{\vec{v}} \\
\vec{v}_{-}
\end{array}\right)+\ldots\right. \\
\left.\quad+\int_{P_{-}}^{S_{g^{-}}}\left(\begin{array}{l}
\overrightarrow{0} \\
\vec{v}_{-}
\end{array}\right)+\left(\begin{array}{l}
K_{P}^{+}-g^{-} \int_{P}^{P_{+}} \vec{v}_{+} \\
K_{P_{-}^{-}}^{-}
\end{array}\right) \mid \operatorname{diag}\left(\mathbb{B}^{+}, \mathbb{B}^{-}\right)\right) \\
=\theta_{+}\left(\int_{P}^{R_{1}} \vec{v}_{+}+\cdots+\int_{P}^{R_{g^{+}}} \vec{v}_{+}+K_{P}^{+}\right) \theta_{-}\left(\int_{P_{-}}^{S_{1}} \vec{v}_{-}+\cdots+\int_{P_{-}}^{S_{g^{-}}} \vec{v}_{-}+K_{P_{-}}^{-}\right) .
\end{aligned}
$$

Now using the asymptotics for the prime-form and $\sigma$, we see that the numerator of (2.37) (with the just made choice of $Q_{1}, \ldots, Q_{g}$ ) is equivalent to

$$
\begin{aligned}
& \epsilon \theta_{+}\left(\int_{P}^{R_{1}} \vec{v}_{+}+\cdots+\int_{P}^{R_{g^{+}}} \vec{v}_{+}+K_{P}^{+}\right) \theta_{-}\left(\int_{P_{-}}^{S_{1}} \vec{v}_{-}+\cdots+\int_{P_{-}}^{S_{g^{-}}} \vec{v}_{-}+K_{P_{-}}^{-}\right) \\
& \times \prod_{i<j} E_{+}\left(R_{i}, R_{j}\right) \prod_{i<j} E_{-}\left(S_{i}, S_{j}\right) \\
& \times\left\{\prod_{i=1}^{g^{+}} \prod_{j=1}^{g^{-}} E_{+}\left(R_{i}, P_{+}\right) E_{-}\left(S_{j}, P_{-}\right)\right\}[\Delta(s)]^{g^{+} g^{-}} \prod_{i=1}^{g^{+}} \sigma_{+}\left(R_{i}, P\right) \frac{\left\{E_{+}\left(P, P_{+}\right)\right\}^{g^{+} g^{-}}}{\left\{\prod_{j=1}^{g^{+}} E_{+}\left(R_{j}, P_{+}\right)\right\}^{g^{-}}} \\
& \times\left[\sigma_{+}\left(P_{+}, P\right)\right]^{g^{-}}\left[E_{+}\left(P_{+}, P\right)\right]^{\left(g^{-}\right)^{2}}[\Delta(s)]^{g^{-}\left(g^{-}-g^{+}\right)} \prod_{j=1}^{g^{-}} \frac{\sigma_{-}\left(S_{j}, P_{-}\right)}{\left\{E_{-}\left(S_{j}, P_{-}\right)\right\}^{g^{+}}},
\end{aligned}
$$

whereas the denominator of (2.37) is equivalent to

$$
\epsilon\left\{\prod_{i=1}^{g^{+}} E_{+}\left(P, R_{i}\right)\right\}\left[E_{+}\left(P, P_{+}\right)\right]^{g^{-}}\left\{\prod_{j=1}^{g^{-}} E_{-}\left(P_{-}, S_{j}\right)\right\}[\Delta(s)]^{g^{-}} \operatorname{det}\left(v_{i}^{+}\left(R_{j}\right)\right) \operatorname{det}\left(v_{i}^{-}\left(S_{j}\right)\right) \text {. }
$$

So, after rearranging the terms and numerous cancelations, one gets the asymptotics

$$
C(P) \sim \epsilon C_{ \pm}(P) C_{\mp}\left(P_{\mp}\right)\left\{E_{ \pm}\left(P, P_{ \pm}\right)\right\}^{g^{\mp}\left(g^{ \pm}+g^{\mp}-1\right)}\left\{\sigma_{ \pm}\left(P_{ \pm}, P\right)\right\}^{g_{\mp}} \Delta(s)^{\left[g^{\mp}\right]^{2}-g^{\mp}}
$$

if $P \in \mathcal{X}^{ \pm}$.

Remark 4. Let us emphasize that in order to define the vector $K^{P}$ and the Abel map $\mathcal{A}_{P}$ (as well as the prime-form and the left-hand side of expression (2.32) ) one has to introduce the system of cuts on the surface $\mathcal{X}$ in such a way that the integration $\int_{x}^{y} \vec{v}$ is well-defined for any $x, y$ belonging to the surface $\mathcal{X}=\mathcal{X}_{s}$ dissected along the cuts. We choose the usual symplectic basis of homologies $\left\{a_{\alpha}^{ \pm}, b_{\alpha}^{ \pm}\right\}_{\alpha=1, \ldots, g^{ \pm}}$ on $\mathcal{X}^{ \pm}$, take curves representing this basis and dissect the $\mathcal{X}^{ \pm}$along these curves. The resulting dissected surface $\mathcal{X}^{ \pm}$is homeomorphic to a sphere with $g^{ \pm}$holes, whereas the surface $\mathcal{X}_{s}$ dissected along the same curves is homeomorphic to a sphere with $g$ holes. Notice that the boundary of any hole is the trivial cycle $\left(a_{\alpha}^{ \pm}+b_{\alpha}^{ \pm}-a_{\alpha}^{ \pm}-b_{\alpha}^{ \pm}=0\right)$ and, therefore, the $\int_{x}^{y} \overrightarrow{v_{ \pm}}$and $\int_{x}^{y} \vec{v}$ are well-defined on the corresponding dissected surfaces. 
The following lemma immediately follows from the definition of the vector of the Riemann constants and Theorem 1 .

$$
K_{\beta}^{P}=\frac{1}{2}+\frac{\mathbb{B}_{\beta \beta}}{2}-\sum_{\alpha=1, \alpha \neq \beta}^{g} \int_{a_{\alpha}}\left(v_{\alpha} \int_{P}^{x} v_{\beta}\right) .
$$

Lemma 3. One has the asymptotics

$$
K^{P} \sim\left(\begin{array}{l}
K_{+}^{P}-g^{-} \int_{P}^{P+} \vec{v}_{+} \\
K_{-}^{P_{-}}
\end{array}\right),
$$

as $s \rightarrow 0$, where $K_{+}^{P}$ and $K_{-}^{P_{-}}$are the vectors of Riemann constants for the surfaces $\mathcal{X}^{+}$and $\mathcal{X}^{-}$with the base points $P$ and $P_{-}$respectively.

2.4. Asymptotics of $\tau_{g}$. Now we are able to prove the asymptotics (1.10) from the Introduction. Let $M_{ \pm}=2 g^{ \pm}-2$ and let $\left(\omega^{ \pm}\right)=\sum_{k=1}^{M_{ \pm}} D_{k}^{ \pm}$be the divisor of the holomorphic differential $\omega^{ \pm}$on $\mathcal{X}^{ \pm}$.

Assume that the point $P$ lies on the component $\mathcal{X}^{+}$. Using Lemma 3, one can pass to the limit $s \rightarrow 0$ in the equation (1.6). This results in the relations

$$
\mathcal{A}_{P}^{+}\left(\left(\omega^{+}\right)\right)=2 K_{+}^{P}+\mathbb{B}^{+} \mathbf{r}^{+}+\mathbf{q}^{+}
$$

and

$$
\mathcal{A}_{P_{-}}^{-}\left(\left(\omega^{-}\right)\right)=2 K_{-}^{P_{-}}+\mathbb{B}^{-} \mathbf{r}^{-}+\mathbf{q}^{+}
$$

where $\mathbf{r}=\left(\mathbf{r}^{+}, \mathbf{r}^{-}\right), \mathbf{q}=\left(\mathbf{q}^{+}, \mathbf{q}^{-}\right)$and $\mathcal{A}^{ \pm}$is the Abel map on $\mathcal{X}^{ \pm}$.

Now one has

$$
\begin{gathered}
\tau^{-6}(\mathcal{X}, z) \sim \epsilon e^{2 \pi i\left\langle\mathbf{r}^{+}, K_{+}^{P}\right\rangle} e^{2 \pi i\left\langle\mathbf{r}^{-}, K_{-}^{P_{-}}\right\rangle} e^{-2 \pi i g^{-}\left\langle\mathbf{r}^{+}, \int_{P}^{P_{+}} \vec{v}_{+}\right\rangle} \\
\times\left\{C_{+}(P)\right\}^{-4}\left\{C_{-}\left(P_{-}\right)\right\}^{-4}\left\{E_{+}\left(P, P_{+}\right)\right\}^{4 g^{-}(1-g)}\left\{\sigma_{+}\left(P_{+}, P\right)\right\}^{-4 g^{-}}[\Delta(s)]^{4\left(g^{-}-\left(g^{-}\right)^{2}\right)} \\
\times \prod_{k=1}^{M_{+}} \sigma_{+}\left(D_{k}^{+}, P\right)\left[\frac{E_{+}\left(P, P_{+}\right)}{E_{+}\left(D_{k}^{+}, P_{+}\right)}\right]^{g^{-}} \\
\times\left[\sigma_{+}\left(P_{+}, P\right)\left\{E_{+}\left(P_{+}, P\right)\right\}^{g^{-}}[\Delta(s)]^{\left(3 g^{-}-g^{+}\right) / 4}\right]^{2} \\
\times \prod_{k=1}^{M_{-}}\left\{\sigma_{-}\left(D_{k}^{-}, P_{-}\right) \sigma_{+}\left(P_{+}, P\right) \frac{\left[E_{+}\left(P_{+}, P\right)\right]^{g^{-}}}{\left[E_{-}\left(D_{k}^{-}, P_{-}\right)\right]^{g^{+}}}[\Delta(s)]^{g^{-}-g^{+}}\right\} \\
\times \prod_{k=1}^{M_{+}}\left\{E_{+}\left(D_{k}^{+}, P\right)\right\}^{(g-1)}\left[[\Delta(s)]^{1 / 4} E\left(P, P_{+}\right)\right]^{2(g-1)} \\
\times \prod_{k=1}^{M_{-}}\left\{\Delta(s) E_{+}\left(P, P_{+}\right) E_{-}\left(D_{k}^{-}, P_{-}\right)\right\}^{(g-1)}
\end{gathered}
$$

with $g=g^{+}+g^{-}$. Observe that $\Delta(s)$ enters the above expression with power $4\left(g^{-}-\left(g^{-}\right)^{2}\right)+\frac{3 g^{-}-g^{+}}{2}+\left(g^{-}-g^{+}\right)\left(2 g^{-}-2\right)+\frac{g-1}{2}+(g-1)\left(2 g^{-}-2\right)=\frac{3}{2}$, all the factors $E_{+}\left(P, P_{+}\right)$cancel out $\left(4 g^{-}(1-g)+g^{-}\left(2 g^{+}-2\right)+2 g^{-}+g^{-}\left(2 g^{-}-\right.\right.$ $\left.2)+2(g-1)+(g-1)\left(2 g^{-}-2\right)=0\right)$ and the remaining terms can be rearranged 
into the product of

$$
\begin{aligned}
& e^{2 \pi i\left\langle\mathbf{r}^{+}, K_{+}^{P}\right\rangle} C_{+}^{-4}(P) \prod_{k=1}^{M_{+}} \sigma_{+}\left(D_{k}^{+}, P\right)\left\{E_{+}\left(D_{k}^{+}, P\right)\right\}^{\left(g^{+}-1\right)}, \\
& e^{2 \pi i\left\langle\mathbf{r}^{-}, K_{-}^{P_{-}}\right\rangle} C_{-}^{-4}\left(P_{-}\right) \prod_{k=1}^{M_{-}} \sigma_{-}\left(D_{k}^{-}, P_{-}\right)\left\{E_{-}\left(D_{k}^{-}, P_{-}\right)\right\}^{\left(g^{-}-1\right)},
\end{aligned}
$$

and

$$
e^{-2 \pi i g^{-}\left\langle\mathbf{r}^{+}, \int_{P}^{P_{+}} \vec{v}_{+}\right\rangle}\left\{\left[\sigma_{+}\left(P_{+}, P\right)\right]^{-2} \frac{\prod_{k=1}^{M_{+}} E_{+}\left(D_{k}^{+}, P\right)}{\prod_{k=1}^{M_{+}} E_{+}\left(D_{k}^{+}, P_{+}\right)}\right\}^{g^{-}} .
$$

According to 11] (see Theorem 2 on page 47), the expression in the braces in (2.48) is nothing but $e^{2 \pi i\left\langle\mathbf{r}^{+}, \mathcal{A}_{P}^{+}\left(P_{+}\right)\right\rangle}$and, therefore, the expression (2.48) equals one; expressions (2.46) and (2.47) coincide with $\tau_{g^{+}}^{-6}\left(\mathcal{X}^{+}, \omega^{+},\left\{a_{\alpha}^{+}, b_{\alpha}^{+}\right\}\right)$and $\tau_{g^{-}}^{-6}\left(\mathcal{X}^{-}, \omega^{-}\right.$, $\left.\left\{a_{\alpha}^{-}, b_{\alpha}^{-}\right\}\right)$respectively.

\section{Surgery AND ASYMPtotics}

3.1. Wentworth lemma. The following important lemma essentially coincides with the statement proved in $\S 3$ of $[22$. We formulate the Wentworth result, adapting it for our needs.

Lemma 4. Let $\mathcal{X}$ be a translation surface, $\Delta$ the Friedrichs extension of the Laplacian on $\mathcal{X}$. Let $z$ be a local parameter near a (nonsingular) point $P \in \mathcal{X}$ such that $z(P)=0$ and $\Delta=-4 \partial_{z} \partial_{\bar{z}}$ in the unit ball $B(1)=\{|z| \leq 1\} \subset \mathcal{X}$. For $0<\epsilon \leq 1$, set $B(\epsilon)=\{|z| \leq \epsilon\}$ and $\mathcal{X}_{\epsilon}=\mathcal{X} \backslash B(\epsilon)$. Denote by $\mathcal{N}_{\epsilon}$ the Dirichlet-to-Neumann operator for $\mathcal{X}_{\epsilon}$ :

$$
\begin{gathered}
\mathcal{N}_{\epsilon}: C^{\infty}\left(\partial \mathcal{X}_{\epsilon}\right) \rightarrow C^{\infty}\left(\partial \mathcal{X}_{\epsilon}\right), \\
\mathcal{N}_{\epsilon}(f)=\left.\partial_{n} u\right|_{\partial \mathcal{X}_{\epsilon}},
\end{gathered}
$$

where the function $u$ satisfies

$$
\left\{\begin{array}{l}
\Delta u=0 \text { in } \mathcal{X}_{\epsilon} \\
\left.u\right|_{\partial X_{\epsilon}}=f
\end{array}\right.
$$

and $n$ is the unit outer normal to $\partial \mathcal{X}_{\epsilon}$. (Actually, $\mathcal{N}_{\epsilon}$ is a pseudodifferential operator of order 1 on $\partial \mathcal{X}_{\epsilon}$.)

Let $z=r e^{i \phi}$. Then $\phi$ is the angular coordinate on the circle $\{r=\epsilon\}=\partial X_{\epsilon}$. Let $f \in L_{2}\left(\partial X_{\epsilon}, d \phi\right), f(\phi)=\sum_{k \in \mathbb{Z}} a_{k} e^{i k \phi}$. Define the (unbounded) operators $\nu$ and $|\nu|$ in $L_{2}\left(\partial X_{\epsilon}, d \phi\right)$ via

$$
\nu f(\phi)=\sum_{k \in \mathbb{Z}} k a_{k} e^{i k \phi}
$$

and

$$
|\nu| f(\phi)=\sum_{k \in \mathbb{Z}}|k| a_{k} e^{i k \phi} .
$$

Then one has the following relation:

$$
\epsilon \mathcal{N}_{\epsilon}=|\nu|+\mathbf{O}(\epsilon)
$$


where $\mathbf{O}(\epsilon)$ is the operator of trace class in $L_{2}\left(\partial \mathcal{X}_{\epsilon}, d \phi\right)$ with the trace norm which is asymptotically $O(\epsilon)$ as $\epsilon \rightarrow 0$.

For completeness we give the proof here (it differs from the one given in 22 by insignificant changes). Introduce the operator $R_{\epsilon}: L_{2}\left(S^{1}, d \phi\right) \rightarrow L_{2}\left(S^{1}, d \phi\right)$ via $R_{\epsilon} f=g=\left.u\right|_{\{|z|=1\}}$, where $u$ and $f$ are from (3.1). Using Green's formula for the Friedrichs extension of the Laplacian, it is easy to check the identity

$$
\int_{|z|=\epsilon}|f|^{2} d \phi-\int_{|z|=1}|g|^{2} d \phi=2 \int_{\epsilon}^{1} \frac{d r}{r} \iint_{\mathcal{X}_{r}}|\nabla u|^{2},
$$

which implies the norm estimate

$$
\left\|R_{\epsilon}\right\| \leq 1
$$

(It is important here that $\Delta$ is the Friedrichs extension; for other extensions the above double integral may be infinite!)

The function $u$ from (3.1) is harmonic in the annulus $\{\epsilon \leq|z| \leq 1\}$ and, therefore, admits there the standard representation

$$
u(r, \phi)=\sum_{k \in \mathbb{Z}} a_{k} e^{i k \phi} r^{k}+c_{0} \log r+\sum_{k \in \mathbb{Z} \backslash 0}\left(r^{|k|}-r^{-|k|}\right) b_{k} e^{i k \phi} .
$$

On the other hand the Green formula implies the relation $\int_{\partial \mathcal{X}_{\epsilon}} \frac{\partial u}{\partial r}=0$ and, therefore, one has $c_{0}=0$ in the previous representation. Thus, the function $u$ from (3.1) is representable inside the annulus $\{\epsilon \leq|z| \leq 1\}$ as

$$
u=a_{0}+\sum_{k \neq 0} a_{k} r^{k} e^{i k \phi}+\sum_{k \neq 0} b_{k} r^{-k} e^{i k \phi} .
$$

Now notice that the operators $\epsilon \mathcal{N}_{\epsilon}$ and $R_{\epsilon}$ map the boundary value of the function $u$ from (3.1) and (3.4) at the circle $|z|=\epsilon$ to the functions

$$
\sum_{k \neq 0} k\left(b_{k} \epsilon^{-k}-a_{k} \epsilon^{k}\right) e^{i k \phi}
$$

and

$$
a_{0}+\sum_{k \neq 0}\left(a_{k}+b_{k}\right) e^{i k \phi}
$$

respectively. For a sequence of complex numbers $\left\{\alpha_{k}\right\}_{k \in \mathbb{Z}}$, introduce the operator (may be unbounded) $\mathrm{Op}\left(\alpha_{k}\right)$ in $L_{2}\left(S^{1}, d \phi\right)$ via

$$
\operatorname{Op}\left(\alpha_{k}\right) f(\phi)=\sum_{k \in \mathbb{Z}} \alpha_{k} a_{k} e^{i k \phi},
$$

where $f \in L_{2}\left(S^{1}, d \phi\right), u=\sum_{k \in \mathbb{Z}} a_{k} e^{i k \phi}$. (In this notation, $\nu=\operatorname{Op}(k)$ and $|\nu|=$ $\mathrm{Op}(|k|)$.

Now one has

$$
\begin{gathered}
\operatorname{Op}\left(\epsilon^{k}-\epsilon^{-k}\right)\left(\epsilon \mathcal{N}_{\epsilon}\right)\left(\left.u\right|_{|z|=\epsilon}\right)=\sum_{k \neq 0} k\left(b_{k}+a_{k}-a_{k} \epsilon^{2 k}-b_{k} \epsilon^{-2 k}\right) e^{i k \phi} \\
=\sum_{k \neq 0}\left(k a_{k}+k b_{k}\right) e^{i k \phi}-\sum_{k \neq o}\left\{k\left(a_{k} \epsilon^{k}+b_{k} \epsilon^{-k}\right)\left(\epsilon^{k}+\epsilon^{-k}\right)-k\left(a_{k}+b_{k}\right)\right\} e^{i k \phi} \\
=2 \sum_{k \neq 0}\left(k a_{k}+k b_{k}\right) e^{i k \phi}-\sum_{k \neq 0}\left(\epsilon^{k}+\epsilon^{-k}\right) k\left(a_{k} \epsilon^{k}+b_{k} \epsilon^{-k}\right) e^{i k \phi},
\end{gathered}
$$


which implies the relation

$$
\epsilon \mathcal{N}_{\epsilon}=\operatorname{Op}\left(\frac{2}{\epsilon^{k}-\epsilon^{-k}}\right) \nu R_{\epsilon}-\mathrm{Op}\left(\frac{\epsilon^{k}+\epsilon^{-k}}{\epsilon^{k}-\epsilon^{-k}}\right) \nu .
$$

(Notice that functions from the image of the operator $\nu$ are orthogonal to 1 and, therefore, the right-hand side of (3.5) is correctly defined.) Clearly, the operator $\operatorname{Op}\left(\frac{2}{\epsilon^{k}-\epsilon^{-k}}\right) \nu$ is of trace class with the trace norm $\left\|\mid \mathrm{Op}\left(\frac{2}{\epsilon^{k}-\epsilon^{-k}}\right) \nu\right\|=O(\epsilon)$; due to (3.3), the same is true for the first term on the right-hand side of (3.5). For $k \neq 0$ one has $\frac{\epsilon^{k}+\epsilon^{-k}}{\epsilon^{k}-\epsilon^{-k}} \rightarrow-$ sgn $k$ as $\epsilon \rightarrow 0$, and a simple estimate shows that

$$
-\mathrm{Op}\left(\frac{\epsilon^{k}+\epsilon^{-k}}{\epsilon^{k}-\epsilon^{-k}}\right) \nu=|\nu|+r(\epsilon)
$$

where $\||r(\epsilon)|\|=O\left(\epsilon^{2}\right)$, which proves the lemma.

3.2. Analytic surgery for translation surfaces. The following proposition is a variant of Theorem $B^{*}$ from [3]. The presence of conical points does not lead to significant difficulties, and the proof remains essentially the same (see discussion in [12]; one can also find a proof of a more general result in [15]).

Proposition 2. Let $\Gamma$ be a smooth closed curve on a translation surface $\mathcal{X}$ containing no conical points and dividing $\mathcal{X}$ into two parts $\mathcal{X}_{1}$ and $\mathcal{X}_{2}$ with common boundary $\Gamma$. Let $\left(\Delta, \mathcal{X}_{1,2}\right)$ be the operators of the Dirichlet boundary value problems in $\mathcal{X}_{1,2}$. Then one has the relation

$$
\operatorname{det}^{*} \Delta=\frac{\operatorname{Area}(X)}{\operatorname{length}(\Gamma)} \operatorname{det}\left(\Delta, \mathcal{X}_{1}\right) \operatorname{det}\left(\Delta, \mathcal{X}_{2}\right) \operatorname{det}^{*}\left(\mathcal{N}_{1}+\mathcal{N}_{2}\right),
$$

where $\mathcal{N}_{k}$ is the Dirichlet-to-Neumann operator $C^{\infty}(\Gamma) \rightarrow \mathbf{C}^{\infty}(\Gamma), \mathcal{N}_{k}(f)=$ $\left.\partial_{n_{k}} u_{k}\right|_{\Gamma}$ with $\Delta u_{k}=0$ in $\mathcal{X}_{k},\left.u\right|_{\Gamma}=f$ and $n_{k}$ being the outer unit normal to $\partial \mathcal{X}_{k}, k=1,2$.

The following proposition (see [22]) is a consequence of the Wentworth lemma.

Proposition 3. Let $\mathcal{X}_{1}=\mathcal{X} \backslash B(\epsilon), \mathcal{X}_{2}=B(\epsilon), \Gamma=\{|z|=\epsilon\}$. Then

$$
\lim _{\epsilon \rightarrow 0} \frac{\operatorname{det}^{*}\left(\mathcal{N}_{1}+\mathcal{N}_{2}\right)}{\operatorname{length}(\Gamma)}=\frac{1}{2} \text {. }
$$

We give a proof of this proposition following [22]. Representing the function $u_{2}$ harmonic in the disk $\mathcal{X}_{2}=\{|z| \leq \epsilon\}$ in the form

$$
u_{2}=\sum_{k \in \mathbb{Z}} a_{k} r^{|k|} e^{i k \phi}
$$

one immediately gets the relation

$$
\epsilon \mathcal{N}_{2}=|\nu|
$$

One now has

$$
\epsilon\left(\mathcal{N}_{1}+\mathcal{N}_{2}\right)=2|\nu|+\mathbf{O}(\epsilon)
$$

and, therefore,

$$
\log \operatorname{det}^{*}\left\{\epsilon\left(\mathcal{N}_{1}+\mathcal{N}_{2}\right)\right\}=\log \operatorname{det}^{*}\{2|\nu|\}+o(1),
$$

which implies that

$$
\log \frac{\operatorname{det}^{*}\left(\mathcal{N}_{1}+\mathcal{N}_{2}\right)}{\epsilon}=\log \frac{\operatorname{det}^{*}|\nu|}{2}+o(1)
$$


or, what is the same,

$$
\log \frac{\operatorname{det}^{*}\left(\mathcal{N}_{1}+\mathcal{N}_{2}\right)}{2 \pi \epsilon}=-\log 2+\log \operatorname{det}^{*}|\nu|-\log 2 \pi+o(1) .
$$

Using the known properties of the Riemann zeta-function, $-2 \zeta^{\prime}(0)=\log 2 \pi$ and $\zeta(0)=-1 / 2$, one gets the relation $\operatorname{det}^{*}|\nu|=2 \pi$, which (together with (3.10) ) implies (3.6).

Remark 5. The implication $(3.7) \Rightarrow(3.8)$ is a consequence of the following estimate:

$$
\begin{gathered}
\left|\log \operatorname{det}^{*}(2|\nu|+\mathbf{O}(\epsilon))-\log \operatorname{det}^{*}(2|\nu|)\right|=\left|\int_{0}^{1} \frac{d}{d t} \log \operatorname{det}^{*}(2|\nu|+t \mathbf{O}(\epsilon)) d t\right| \\
\leq \int_{0}^{1}\left|\operatorname{tr}\left(\left.(2|\nu|+t \mathbf{O}(\epsilon))\right|_{\{1\}^{\perp}} ^{-1} \mathbf{O}(\epsilon)\right)\right| d t \leq C_{1}|\operatorname{tr} \mathbf{O}(\epsilon)| \leq C_{2} \epsilon
\end{gathered}
$$

(cf. 14], Lemma 4.1).

Remark 6. The implication (3.8) $\Rightarrow(3.9)$ is a consequence of the standard relations

$$
\begin{gathered}
\operatorname{det}^{*}(\epsilon A)=\epsilon^{\zeta_{A}(0)} \operatorname{det}^{*} A \\
\zeta_{\mathcal{N}_{1}+\mathcal{N}_{2}}(0)=\mathbf{h}_{0}-\operatorname{dim} \operatorname{Ker}\left(\mathcal{N}_{1}+\mathcal{N}_{2}\right)=\mathbf{h}_{0}-1,
\end{gathered}
$$

where $\mathbf{h}_{0}$ is the constant term in the asymptotical expansion of $\operatorname{tr} e^{-t\left(\mathcal{N}_{1}+\mathcal{N}_{2}\right)}$ as $t \rightarrow 0+$, the relation $\zeta_{|\nu|}(0)=-1$ and the result from [5]:

$$
\mathbf{h}_{0}=0 \text {. }
$$

Corollary 1. One has the asymptotics

$$
\operatorname{det}(\Delta, \mathcal{X} \backslash B(\epsilon)) \sim \frac{2^{7 / 6} \sqrt{\pi} e^{2 \zeta^{\prime}(-1)+5 / 12} \operatorname{det}^{*} \Delta}{\operatorname{Area}(\mathcal{X})} \epsilon^{1 / 3}
$$

as $\epsilon \rightarrow 0$.

Proof. This immediately follows from Proposition 2, (3.6) and the relation

$$
\operatorname{det}(\Delta, B(\epsilon))=2^{-1 / 6} \pi^{-1 / 2} \epsilon^{-1 / 3} e^{-2 \zeta^{\prime}(-1)-5 / 12}
$$

found in [21].

Remark 7. It is interesting to compare (3.14) with the asymptotics of the first eigenvalue of the operator of the Dirichlet boundary value problem in $\mathcal{X} \backslash B(\epsilon)$,

$$
\lambda_{1}(\Delta, \mathcal{X} \backslash B(\epsilon)) \sim-\frac{2 \pi}{\operatorname{Area}(\mathcal{X})}(\log \epsilon)^{-1}
$$

as $\epsilon \rightarrow 0$, which was found in [18].

3.3. Symmetric case. Let $\mathcal{X}$ be a translation surface of genus $g \geq 1, z$ a local coordinate in the vicinity of a nonsingular point $P$ of $\mathcal{X}$, such that $z(P)=0$ and in the ball $\{|z| \leq \epsilon\}$ the operator $\Delta$ acts as $-4 \partial_{z} \partial_{\bar{z}}$. Introduce a straight cut $I(\epsilon / 2)$ connecting the points $z=0$ and $z=\epsilon / 2$ and glue two copies of $\mathcal{X} \backslash I(\epsilon / 2)$ along the cut in a usual way. One gets a translation surface $\widehat{\mathcal{X}}$ of genus $2 g$ and the area $\operatorname{Area}(\widehat{\mathcal{X}})=2 \operatorname{Area}(\mathcal{X})$. The endpoints of the cut give rise to two conical points, $P_{1}, P_{2}$, of conical angles $4 \pi$ on $\widehat{\mathcal{X}}$. Let $\widehat{\Delta}$ be the (Friedrichs extension of) the Laplacian on $\widehat{\mathcal{X}}$. The following statement is a very special case of (1.11) proved in an alternative way in order to get information about the unknown constants $\delta_{g}$ in (1.11). 
Proposition 4. One has the asymptotics

$$
\operatorname{det}^{*} \widehat{\Delta} \sim \frac{2 \kappa_{0}}{\operatorname{Area}(\mathcal{X})}\left\{\operatorname{det}^{*} \Delta\right\}^{2} \epsilon^{1 / 2},
$$

as $\epsilon \rightarrow 0$, where the constant $\kappa_{0}$ is the same for all translation surfaces $\mathcal{X}$ (and for all $g \geq 1$ ) and is defined via formula (3.27) below.

Remark 8. The factor $2 / \operatorname{Area}(\mathcal{X})$ is nothing but $\operatorname{Area}(\widehat{\mathcal{X}}) /[\operatorname{Area}(\mathcal{X}) \operatorname{Area}(\mathcal{X})]$. That is why we are not attaching the factor 2 to the constant $\kappa_{0}$ in (3.15).

Proof. First, notice that the surface $\widehat{\mathcal{X}}$ is provided with a natural involution $*$, and the shores of the cut $I(\epsilon)$ (two homologous saddle connections, $\gamma$ and $\gamma^{\prime}$ on $\widehat{\mathcal{X}}$ ) are fixed by this involution. One has the standard ( $\widehat{\Delta}$-invariant) decomposition $L_{2}(\widehat{\mathcal{X}})=L_{2}^{\text {symm }}(\widehat{\mathcal{X}}) \oplus L_{2}^{\text {antisymm }}(\widehat{\mathcal{X}})$ and the functions $u$ from the domain of $\widehat{\Delta}$ which enter $L_{2}^{\text {antisymm }}(\widehat{\mathcal{X}})$ satisfy $\left.u\right|_{\gamma \cup \gamma^{\prime}}=0$, whereas the functions $u$ from the domain of $\widehat{\Delta}$ which enter $L_{2}^{\text {symm }}(\widehat{\mathcal{X}})$ satisfy $\left.u_{n}\right|_{\gamma \cup \gamma^{\prime}}=0$. This shows that the operator $\widehat{\Delta}$ is unitarily equivalent to the direct sum of the operators $\Delta_{\mathcal{D}}$ and $\Delta_{\mathcal{N}}$ of the homogeneous Dirichlet and Neumann boundary value problems in $\mathcal{X} \backslash I(\epsilon)$ (cf., e.g., 9], p. 79) and, therefore,

$$
\operatorname{det}^{*} \widehat{\Delta}=\operatorname{det} \Delta_{\mathcal{D}} \operatorname{det}^{*} \Delta_{\mathcal{N}}
$$

We are to study the asymptotics of $\operatorname{det} \Delta_{\mathcal{D}}$ and $\operatorname{det}^{*} \Delta_{\mathcal{N}}$ as $\epsilon \rightarrow 0$.

Asymptotics of $\operatorname{det} \Delta_{\mathcal{D}}$. We will be using the generalizations of the BFK formula (Theorems B and $\mathrm{B}^{*}$ from [3]) to the case of Laplacians on $2 d$ manifolds with boundary with Dirichlet (and Neumann) boundary conditions. Such generalizations are straightforward and are mentioned in 14. (see Remark on page 326). Their proofs differ from the standard proof of Theorem $B^{*}$ from [3] insignificantly. For the operator $\Delta_{\mathcal{D}}$ the following surgery formula holds true:

$$
\operatorname{det} \Delta_{\mathcal{D}}=\operatorname{det}(\Delta, \mathcal{X} \backslash B(\epsilon)) \operatorname{det}(\Delta, B(\epsilon) \backslash I(\epsilon)) \operatorname{det}\left(\mathcal{N}_{\epsilon}+\mathcal{N}_{\epsilon}^{\text {int }, D}\right),
$$

where $\mathcal{N}_{\epsilon}$ is the Dirichlet-to-Neumann operator from Lemma 4 the operator $\mathcal{N}_{\epsilon}^{\text {int,D }}: C^{\infty}(\partial B(\epsilon)) \rightarrow C^{\infty}(\partial B(\epsilon))$ is defined via $\mathcal{N}_{\epsilon}^{\text {int }, D}(f)=\left.u_{n}\right|_{\partial B(\epsilon)}$, with $u$ subject to

$$
\left\{\begin{array}{l}
\Delta u=0 \text { in } B(\epsilon) \backslash I(\epsilon) \\
\left.u\right|_{\partial B(\epsilon)}=f \\
\left.u\right|_{I(\epsilon)}=0
\end{array}\right.
$$

and $(\Delta, B(\epsilon) \backslash I(\epsilon))$ is the operator of the homogeneous Dirichlet boundary value problem in $B(\epsilon) \backslash I(\epsilon)$. (Notice that there are no coefficients of the type Area/length on the right-hand side of (3.17): all the operators there are invertible and (3.17) is an analog of Theorem B from [3].) The asymptotic formula for the first factor in (3.17) is given in (3.14); the asymptotic formulas for the other two factors can be obtained as consequences of homogeneity properties. Due to (3.11) one has

$$
\operatorname{det}(\Delta, B(\epsilon) \backslash I(\epsilon))=\epsilon^{-2 \zeta_{(\Delta, B(\epsilon) \backslash I(\epsilon))}(0)} \operatorname{det}(\Delta, B(1) \backslash I(1)),
$$

where the value of $\zeta_{(\Delta, B(\epsilon) \backslash I(\epsilon))}(0)$ coincides with the term $\mathbf{h}_{0}$ of the corresponding heat asymptotics (cf. (3.12); clearly, $\operatorname{dimKer}(\Delta, B(\epsilon) \backslash I(\epsilon))=0$ ). The term $\mathbf{h}_{0}$ is 
easy to find; namely, one has

$$
\mathbf{h}_{0}=\frac{1}{6}+2 \frac{\pi^{2}-(2 \pi)^{2}}{24 \pi(2 \pi)}=\frac{1}{24},
$$

where the term $\frac{1}{6}$ comes from the part $\partial B(\epsilon)$ of $\partial[B(\epsilon) \backslash I(\epsilon)]$ and two terms $\frac{\pi^{2}-(2 \pi)^{2}}{24 \pi(2 \pi)}$ come from two angle points of opening $\beta=2 \pi$ at the endpoints of the cut $I(\epsilon)$ (see [4, formula (4.41) or 2], formula (37); the straight part, int $(I(\epsilon))$ of the boundary makes no input in $\left.\mathbf{h}_{0}\right)$. Thus, one has

$$
\operatorname{det}(\Delta, B(\epsilon) \backslash I(\epsilon))=\epsilon^{-\frac{1}{12}} \operatorname{det}(\Delta, B(1) \backslash I(1)) .
$$

Moreover, from (3.13) and the relation $\operatorname{dimKer}\left(\mathcal{N}_{\epsilon}+\mathcal{N}_{\epsilon}^{\text {int, }}\right.$ ) $)=0$ one gets the equality

$$
\zeta_{\mathcal{N}_{\epsilon}+\mathcal{N}_{\epsilon}^{\text {int }, D}}(0)=0
$$

and, therefore,

$$
\begin{aligned}
\operatorname{det}\left(\mathcal{N}_{\epsilon}+\mathcal{N}_{\epsilon}^{i n t, D}\right) & =\operatorname{det}\left(\epsilon \mathcal{N}_{\epsilon}+\epsilon \mathcal{N}_{\epsilon}^{i n t, D}\right) \\
& =\operatorname{det}\left(|\nu|+\mathbf{O}(\epsilon)+\mathcal{N}_{1}^{\text {int }, D}\right) \sim \operatorname{det}\left(|\nu|+\mathcal{N}_{1}^{\text {int }, D}\right),
\end{aligned}
$$

as $\epsilon \rightarrow 0$ due to Lemma 4. Summarizing, one arrives at the asymptotic formula

$$
\operatorname{det} \Delta_{\mathcal{D}} \sim \frac{2^{7 / 6} \sqrt{\pi} e^{2 \zeta^{\prime}(-1)+5 / 12} \operatorname{det}^{*} \Delta \operatorname{det}\left(|\nu|+\mathcal{N}_{1}^{i n t, D}\right) \operatorname{det}(\Delta, B(1) \backslash I(1))}{\operatorname{Area}(\mathcal{X})} \epsilon^{\frac{1}{4}}
$$

as $\epsilon \rightarrow 0$.

Asymptotics of $\operatorname{det} \Delta_{\mathcal{N}}$. For the operator $\Delta_{\mathcal{N}}$ the analog of Theorem B* from [3] looks as follows:

$$
\operatorname{det}^{*} \Delta_{\mathcal{N}}=\frac{\operatorname{Area}(\mathcal{X})}{2 \pi \epsilon} \operatorname{det}(\Delta, \mathcal{X} \backslash B(\epsilon)) \operatorname{det}(\Delta, B(\epsilon) \backslash I(\epsilon) ; D, N) \operatorname{det}^{*}\left(\mathcal{N}_{\epsilon}+\mathcal{N}_{\epsilon}^{\text {int }, N}\right),
$$

where $\mathcal{N}_{\epsilon}$ is the Dirichlet-to-Neumann operator from Lemma 4 the operator $\mathcal{N}_{\epsilon}^{\text {int,N }}: C^{\infty}(\partial B(\epsilon)) \rightarrow C^{\infty}(\partial B(\epsilon))$ is defined via $\mathcal{N}_{\epsilon}^{\text {int }, N}(f)=\left.u_{n}\right|_{\partial B(\epsilon)}$, with $u$ subject to

$$
\left\{\begin{array}{l}
\Delta u=0 \text { in } B(\epsilon) \backslash I(\epsilon) \\
\left.u\right|_{\partial B(\epsilon)}=f \\
\left.u_{n}\right|_{I(\epsilon)}=0
\end{array}\right.
$$

and $(\Delta, B(\epsilon) \backslash I(\epsilon) ; D, N)$ is the operator of the homogeneous boundary value problem in $B(\epsilon) \backslash I(\epsilon)$ with Dirichlet conditions on $\partial B(\epsilon)$ and Neumann conditions on $I(\epsilon)$. As above, the asymptotic formula of the first factor in (3.24) is given in (3.14); the asymptotic formulas of the other two factors can be obtained as consequences of homogeneity properties. Due to (3.11) one has

$$
\begin{aligned}
\operatorname{det}(\Delta, B(\epsilon) \backslash I(\epsilon)) & =\epsilon^{-2 \zeta_{(\Delta, B(\epsilon) \backslash I(\epsilon) ; D, N)}(0)} \operatorname{det}(\Delta, B(1) \backslash I(1) ; D, N) \\
& =\epsilon^{-\frac{1}{12}} \operatorname{det}(\Delta, B(1) \backslash I(1) ; D, N) .
\end{aligned}
$$

(The inputs from the angle points to the $\mathbf{h}_{0}$ are the same for Dirichlet and Neumann problems.) Since $\operatorname{dim} \operatorname{Ker}\left(\mathcal{N}_{\epsilon}+\mathcal{N}_{\epsilon}^{\text {int, }},{ }^{2}\right)=1$, one gets the equality

$$
\zeta_{\mathcal{N}_{\epsilon}+\mathcal{N}_{\epsilon}^{i n t, N}}(0)=-1
$$


and, therefore,

$$
\begin{aligned}
\operatorname{det}^{*}\left(\mathcal{N}_{\epsilon}+\mathcal{N}_{\epsilon}^{i n t, N}\right) & =\epsilon \operatorname{det}^{*}\left(\epsilon \mathcal{N}_{\epsilon}+\epsilon \mathcal{N}_{\epsilon}^{\text {int }, N}\right) \\
& =\epsilon \operatorname{det}^{*}\left(|\nu|+\mathbf{O}(\epsilon)+\mathcal{N}_{1}^{\text {int }, N}\right) \sim \epsilon \operatorname{det}^{*}\left(|\nu|+\mathcal{N}_{1}^{\text {int }, N}\right),
\end{aligned}
$$

as $\epsilon \rightarrow 0$ due to Lemma 4. Summarizing, one arrives at the asymptotic formula

$\operatorname{det}^{*} \Delta_{\mathcal{N}} \sim 2^{1 / 6} \pi^{-1 / 2} e^{2 \zeta^{\prime}(-1)+5 / 12} \operatorname{det}^{*} \Delta \operatorname{det}^{*}\left(|\nu|+\mathcal{N}_{1}^{i n t, N}\right) \operatorname{det}(\Delta, B(1) \backslash I(1) ; D, N) \epsilon^{\frac{1}{4}}$

as $\epsilon \rightarrow 0$. Now from (3.26), (3.22) and (3.16) one gets (3.15) with

$$
\begin{aligned}
\kappa_{0}= & 2^{1 / 3} e^{4 \zeta^{\prime}(-1)+5 / 6} \operatorname{det}\left(|\nu|+\mathcal{N}_{1}^{i n t, D}\right) \operatorname{det}(\Delta, B(1) \backslash I(1)) \operatorname{det}^{*}\left(|\nu|+\mathcal{N}_{1}^{i n t, N}\right) \\
& \times \operatorname{det}(\Delta, B(1) \backslash I(1) ; D, N) .
\end{aligned}
$$

Now from (1.11) (with $s=\epsilon / 2$ ) and (3.15) one gets the relation

$$
\delta_{2 g}=2 \sqrt{2} \kappa_{0}\left(\delta_{g}\right)^{2}
$$

for the constant $\delta_{g}$ from (1.7). This implies that

$$
\delta_{N}=\left(2 \sqrt{2} \kappa_{0}\right)^{N-1} \delta_{1}^{N},
$$

with $\delta_{1}$ from (1.9) for any $N$ of the form $N=2^{n}$. In the next subsection we show that (3.28) holds for any natural number $N$.

3.4. General case. Let $\mathcal{Y}$ be a translation surface of genus $g-1$ and also let $T$ be a translation surface of genus one (a flat torus). Take two disks, $D_{\mathcal{Y}}$ and $D_{T}$, of radius $\epsilon$ in $\mathcal{Y}$ and $T$ with centers $P$ and $Q$ and introduce two straight cuts of length $\epsilon / 2$ starting at points $P \in \mathcal{Y}$ and $Q \in T$. Gluing the surfaces $\mathcal{Y}$ and $T$ along the cuts one gets the translation surface $\mathcal{X}$ of genus $g$. The shores of the cuts give rise to the saddle connections $\gamma$ and $\gamma^{\prime}$ on $\mathcal{X}$. The boundary $\partial B(\epsilon)=\partial D_{\mathcal{Y}}$ of the disk in $\mathcal{Y}$ gives rise to the contour $\Gamma$ on $\mathcal{X}$. Let $\mathcal{Y}_{\epsilon}=\mathcal{Y} \backslash D_{\mathcal{Y}}$ and $\mathcal{Z}_{\epsilon}=\mathcal{X} \backslash \mathcal{Y}_{\epsilon}$. Let $\mathcal{W}_{\epsilon}=T \backslash D_{T}$. Gluing $\mathcal{W}_{\epsilon}$ and $\mathcal{Z}_{\epsilon}$ along the boundary $\partial D_{T}=\Gamma$ one gets the symmetric translation surface $S$ of genus two.

By virtue of Proposition 2, one has

$$
\begin{aligned}
\operatorname{det}^{*} \Delta_{\mathcal{X}} & =\frac{\operatorname{Area}(\mathcal{X})}{\operatorname{length}(\Gamma)} \operatorname{det}\left(\Delta, \mathcal{Y}_{\epsilon}\right) \operatorname{det}\left(\Delta, \mathcal{Z}_{\epsilon}\right) \operatorname{det}^{*}\left(\mathcal{N}_{\mathcal{Y}_{\epsilon}}+\mathcal{N}_{\mathcal{Z}_{\epsilon}}\right), \\
\operatorname{det}^{*} \Delta_{S} & =\frac{2 \operatorname{Area}(T)}{\operatorname{length}(\Gamma)} \operatorname{det}\left(\Delta, \mathcal{W}_{\epsilon}\right) \operatorname{det}\left(\Delta, \mathcal{Z}_{\epsilon}\right) \operatorname{det}^{*}\left(\mathcal{N}_{\mathcal{W}_{\epsilon}}+\mathcal{N}_{\mathcal{Z}_{\epsilon}}\right)
\end{aligned}
$$

and, therefore,

$$
\operatorname{det}^{*} \Delta_{\mathcal{X}}=\frac{\operatorname{Area}(\mathcal{X})}{2 \operatorname{Area}(T)} \frac{\operatorname{det}^{*}\left(\mathcal{N}_{\mathcal{Y}_{\epsilon}}+\mathcal{N}_{\mathcal{Z}_{\epsilon}}\right)}{\operatorname{det}^{*}\left(\mathcal{N}_{\mathcal{W}_{\epsilon}}+\mathcal{N}_{\mathcal{Z}_{\epsilon}}\right)} \frac{\operatorname{det}\left(\Delta, \mathcal{Y}_{\epsilon}\right)}{\operatorname{det}\left(\Delta, \mathcal{W}_{\epsilon}\right)} \operatorname{det}^{*} \Delta_{S}
$$

Using Lemma 4, we have

$$
\begin{gathered}
\log \frac{\operatorname{det}^{*}\left(\mathcal{N}_{\mathcal{Y}_{\epsilon}}+\mathcal{N}_{\mathcal{Z}_{\epsilon}}\right)}{\operatorname{det}^{*}\left(\mathcal{N}_{\mathcal{W}_{\epsilon}}+\mathcal{N}_{\mathcal{Z}_{\epsilon}}\right)}=\log \frac{\operatorname{det}^{*}\left(|\nu|+\mathbf{O}_{1}(\epsilon)+\mathcal{N}_{\mathcal{Z}_{\epsilon}}\right)}{\operatorname{det}^{*}\left(|\nu|+\mathbf{O}_{2}(\epsilon)+\mathcal{N}_{\mathcal{Z}_{\epsilon}}\right)} \\
=\int_{0}^{1} \frac{d}{d t} \log \operatorname{det}^{*}\left(|\nu|+t \mathbf{O}_{1}(\epsilon)+(1-t) \mathbf{O}_{2}(\epsilon)+\mathcal{N}_{\mathcal{Z}_{\epsilon}}\right) d t \\
=\int_{0}^{1} \operatorname{tr}\left[\left(|\nu|+t \mathbf{O}_{1}(\epsilon)+(1-t) \mathbf{O}_{2}(\epsilon)+\mathcal{N}_{\mathcal{Z}_{\epsilon}}\right)_{\{1\}^{\perp}}^{-1}\left(\mathbf{O}_{1}(\epsilon)-\mathbf{O}_{2}(\epsilon)\right)\right] d t=O(\epsilon)
\end{gathered}
$$


and, therefore,

$$
\frac{\operatorname{det}^{*}\left(\mathcal{N}_{\mathcal{Y}_{\epsilon}}+\mathcal{N}_{\mathcal{Z}_{\epsilon}}\right)}{\operatorname{det}^{*}\left(\mathcal{N}_{\mathcal{W}_{\epsilon}}+\mathcal{N}_{\mathcal{Z}_{\epsilon}}\right)} \sim 1
$$

as $\epsilon \rightarrow 0$. Due to (3.14) we get

$$
\frac{\operatorname{det}\left(\Delta, \mathcal{Y}_{\epsilon}\right)}{\operatorname{det}\left(\Delta, \mathcal{W}_{\epsilon}\right)} \sim \frac{\operatorname{det}^{*} \Delta_{\mathcal{Y}}}{\operatorname{det}^{*} \Delta_{T}} \frac{\operatorname{Area}(T)}{\operatorname{Area}(\mathcal{Y})}
$$

Finally, from (3.15) it follows that

$$
\operatorname{det}^{*} \Delta_{S} \sim \frac{2 \kappa_{0}}{\operatorname{Area}(T)}\left\{\operatorname{det}^{*} \Delta_{T}\right\}^{2} \epsilon^{\frac{1}{2}}
$$

Thus, we conclude from (3.31) that

$$
\operatorname{det}^{*} \Delta_{\mathcal{X}} \sim \frac{\operatorname{Area}(\mathcal{X})}{\operatorname{Area}(\mathcal{Y}) \operatorname{Area}(T)} \kappa_{0} \operatorname{det}^{*} \Delta_{\mathcal{Y}} \operatorname{det}^{*} \Delta_{T} \epsilon^{\frac{1}{2}}
$$

as $\epsilon \rightarrow 0$. Comparing (3.32) and (1.11) (with $s:=\epsilon / 2$ ), one arrives at the following proposition.

Proposition 5. The following expression for the constant $\delta_{g}$ in the formula (1.7) for the determinant of the Laplacian on the stratum $H_{g}(1, \ldots, 1)$ holds true:

$$
\delta_{g}=\frac{1}{2 \sqrt{2}}\left(2^{1 / 6} \pi^{-4 / 3}\right)^{g} \kappa_{0}^{g-1},
$$

where $\kappa_{0}$ is given by (3.27).

\section{REFERENCES}

[1] Ahlfors L., Sario L., Riemann surfaces, Princeton University Press, Princeton, NJ 1960. MR0114911 (22:5729)

[2] Aurell E., Salomonson P., On functional determinants of Laplacians in polygons and simplicial complexes, Commun. Math. Phys. 165, 233-259 (1994). MR1301847(95i:58186)

[3] Burghelea D., Friedlander L., Kappeler T., Meyer-Vietoris type formula for determinants of elliptic differential operators, J. Funct. Anal., 107 (1992), N1, 34-65. MR:1165865(93f:58242)

[4] Cheeger, J., Spectral Geometry of singular Riemannian spaces, J. Differential Geom., 18 (1983), 575-657. MR730920 (85d:58083)

[5] Edward J., Wu S., Determinant of the Neumann operator on smooth Jordan curves, Proceedings of the American Mathematical Society, Vol. 111, N2, 1991. MR1031662 (91f:58094)

[6] Eskin A., Masur H., Zorich A., Moduli spaces of Abelian differentials: the principal boundary, counting problems and the Siegel-Veech constants, math.DS/0202134.

[7] Fay, John D., Theta-functions on Riemann surfaces, Lect. Notes in Math., 352, Springer (1973). MR0335789(49:569)

[8] Fay, John D., Kernel functions, analytic torsion, and moduli spaces, Memoirs of the AMS 464 (1992). MR1146600 (93e:58192)

[9] Hillairet L., Contribution d'orbites périodiques diffractives à la formule de trace, Ph.D. Thesis, L'Institut Fourier, Grenoble, 2002.

[10] J. Jorgenson, Asymptotic behavior of Faltings's delta function. Duke Math. J., 61 (1990), N1, 221-254. MR1068387 (91m:14042)

[11] Kokotov A., Korotkin D., Tau-functions on spaces of Abelian differentials and higher genus generalizations of Ray-Singer formula, Journal of Differential Geometry, 82 (2009), 35-100. MR2504770 (2010c:58041)

[12] Kokotov A., Korotkin D., Tau-functions on the spaces of Abelian and quadratic differentials and determinants of Laplacians in Strebel metrics of finite volume, preprint of Max-Planck Institute for Mathematics in the Sciences, Leipzig, 46/2004; math.SP/0405042.

[13] Kontsevich, M., Zorich, A., Connected components of the moduli spaces of holomorphic differentials with prescribed singularities, Invent. Math. 153 631-678 (2003). MR2000471 (2005b:32030) 
[14] Yoonweon Lee, Burghelea-Friedlander-Kappeler's gluing formula for the zeta-determinant and its applications to the adiabatic decompositions of the zeta-determinant and analytic torsion, Transactions of the American Mathematical Society, Vol. 355, N10, pp. 4093-4110. MR.1990576 (2004e:58058)

[15] Loya P., McDonald P., Park J., Zeta regularized determinants for conic manifolds, Journal of Functional Analysis (2007), 242, N1, 195-229. MR2274020 (2007g:58036)

[16] R. Lundelius, Asymptotics of the determinant of the Laplacian on hyperbolic surfaces of finite volume, Duke Math. J. 71 (1993), no. 1, 211-242. MR.1230291(94j:58178)

[17] Masur H., The extension of the Weil-Peterson metric to the boundary of Teichmuller space, Duke Mathematical Journal, Vol. 43(1976), N3, pp. 623-635. MR0417456 (54:5506)

[18] Ozawa S., The first eigenvalue of the Laplacian on two-dimensional Riemannian manifolds, Tohoku Math. Journ., 34(1982), 7-14. MR651702 (83g:58073)

[19] Polchinski J., Evaluation of the one loop string path integral, Commun. Math. Phys., 104 (1986) 37-47. MR834480 (87j:81223)

[20] Ray D. B., Singer I. M., Analytic torsion for complex manifolds. Ann. of Math. (2) 98 154-177 (1973), MR383463, Zbl 0267.32014.

[21] Weisberger W., Conformal Invariants for determinants of Laplacians on Riemann surfaces, Commun. Math. Phys., 112, 633-638 (1987). MR910583 (89c:58135)

[22] Wentworth R., Precise constants in bosonization formulas on Riemann surfaces, Commun. Math. Phys. 282 (2) (2008), 339-355. MR2421480(2009f:58054)

[23] Wentworth R., private communication.

[24] Wentworth R., Asymptotics of determinants from functional integration, J. Math. Phys., 32(7), 1991, 1767-1773. MR1112704 (92k:58271)

[25] Wentworth R., The asymptotics of the Arakelov-Green's function and Faltings' delta invariant, Commun. Math. Phys. 137, (1991), 427-459. MR1105425 (92g:14019)

[26] Wolpert S., Asymptotics of the spectrum and the Selberg zeta function on the space of Riemann surfaces, Comm. Math. Phys. 112 (1987), no. 2, 283-315. MR905169 (89c:58136)

[27] Yamada A., Precise variational formulas for abelian differentials, Kodai Math. J., 3 (1980), 114-143. MR569537 (81i:30078)

Department of Mathematics and Statistics, Concordia University, 1455 de Maisonneuve Blvd. West, Montreal, Quebec, Canada H3G 1M8

E-mail address: alexey@mathstat.concordia.ca 